\title{
Development of A Novel Anti-Liver Fibrosis Formula with luteolin, licochalcone $A$, aloe-emodin and acacetin by network pharmacology and transcriptomics analysis
}

\section{Yuan Zhou}

Shanghai University of TCM: Shanghai University of Traditional Chinese Medicine

\section{Rong Wu}

Shanghai University of TCM: Shanghai University of Traditional Chinese Medicine

\section{Fei-Fei Cai}

Shanghai University of TCM: Shanghai University of Traditional Chinese Medicine

Wen-Jun Zhou

Shanghai University of TCM: Shanghai University of Traditional Chinese Medicine Yi-Yu Lu

Shanghai University of TCM: Shanghai University of Traditional Chinese Medicine

Hui Zhang

Shanghai University of TCM: Shanghai University of Traditional Chinese Medicine

\section{Qi-Long Chen}

Shanghai University of TCM: Shanghai University of Traditional Chinese Medicine

\section{Mingyu Sun}

Shanghai University of TCM: Shanghai University of Traditional Chinese Medicine

\section{Su Shi-Bing ( $\square$ shibingsu07@163.com )}

Shanghai University of Traditional Chinese Medicine https://orcid.org/0000-0002-1003-6424

\section{Research}

Keywords: Liver fibrosis, Xiaoyaosan decoction, LLAAF, Network pharmacology, Anti-liver fibrotic effect

Posted Date: November 18th, 2020

DOl: https://doi.org/10.21203/rs.3.rs-107308/v1

License: (c) (1) This work is licensed under a Creative Commons Attribution 4.0 International License. Read Full License 
Version of Record: A version of this preprint was published at Pharmaceutical Biology on January 1st, 2021. See the published version at https://doi.org/10.1080/13880209.2021.1999275. 


\title{
Development of A Novel Anti-Liver Fibrosis Formula with luteolin,
}

\section{licochalcone A, aloe-emodin and acacetin by network pharmacology and transcriptomics analysis}

\author{
Author names \\ Yuan Zhou ${ }^{1 \mathrm{a}}$, Rong Wu ${ }^{1 \mathrm{a}}$, Fei-Fei Cai ${ }^{\mathrm{a}}$, Wen-Jun Zhou ${ }^{\mathrm{a}}$, Yi-Yu Lu', Hui Zhang ${ }^{\mathrm{a}}$, Qi-Long Chen ${ }^{\mathrm{a}}$, \\ Mingyu $\operatorname{Sun}^{\mathrm{b}} *$, Shi-Bing $\mathrm{Su}^{\mathrm{a} *}$ \\ Affiliations \\ a Research Center for Traditional Chinese Medicine Complexity System, Shanghai, Institute of \\ Interdisciplinary Integrative Medicine Research, University of Traditional Chinese Medicine, \\ Shanghai, 201203, China \\ b Key Laboratory of Liver and Kidney Diseases (Ministry of Education), Institute of Liver \\ Diseases, Shuguang Hospital, Shanghai University of Traditional Chinese Medicine, \\ Shanghai 200233, China
}

\section{Corresponding author}

*Shi-Bing Su, shibingsu07@163.com; 021-51323013 and Mingyu Sun, mysun248@hotmail.com

${ }^{1}$ These authors have contributed equally to this study.

E-mail addresses: ydazhou@sina.com (Y. Zhou),wurong_31@163.com (R. Wu), huihuicai15@aliyun.com (F.-F. Cai), 715524159@qq.com (W.-J. Zhou), yiyulu@shutcm.edu.cn (Y.-Y. Lu), zhanghuiman@126.com (H. Zhang), cqlw1975@126.com (Q.-L. Chen), mysun248@hotmail.com (M. Sun), shibingsu07@163.com (S.-B.Su). 


\begin{abstract}
:
Background: Liver fibrosis leads to loss of liver function. Xiaoyaosan decoction (XYS) as a classical Traditional Chinese Medicine (TCM) formula is used to treatment liver fibrosis in clinical and alleviated CCl4-induced liver fibrosis in our previous study.

Xiaoyaosan decoction was composed of many ingredients, and the active ingredients is not clear. The Aim of this study is to explore the clarified compounds or compound combinations to treat liver fibrosis.
\end{abstract}

Methods: Network pharmacology combined with transcriptomics analysis were used to analyze the Xiaoyaosan decoction (XYS) and liver depression and spleen deficiency syndrome liver fibrosis. This consisted constructed XYS-Syndrome-liver fibrosis network, the predict formula named LLAAF was develop from the network by topological analysis according to network stability. The anti-fibrosis effect was evaluated by in vitro and in vivo study.

Results: According to the network XYS-Syndrome-liver fibrosis network, luteolin, licochalcone A, aloe-emodin, and acacetin formula (LLAAF) was predicted from 8 key compounds and 255 combinations in XYS, and LLAAF had a synergistic effect on the proliferation inhibition of hepatic stellate cells (HSCs) compared to each individual compound alone. The treatment of XYS and LLAAF showed a similar anti-liver fibrotic effect that reduced histopathological changes of liver fibrosis, Hyp content and levels of $\alpha$-SMA and collagen I in $\mathrm{CCl}_{4}$-induced liver fibrosis in rat. Transcriptomics analysis revealed LLAAF regulated PI3K-Akt, AMPK, FoxO, Jak-STAT3, P53, cell cycle, focal adhesion, and PPAR signaling. Furthermore, LLAAF was confirmed to regulate Jak-STAT and PI3K-Akt-FoxO signaling in vitro and in vivo. 
Conclusions: This study developed a novel anti-liver formula, LLAAF from XYS demonstrated the anti-liver fibrotic activity may be involved in the regulation of Jak-STAT and PI3K-AktFoxO signaling. 
Keywords: Liver fibrosis; Xiaoyaosan decoction; LLAAF; Network pharmacology; Anti-liver fibrotic effect 


\section{Background}

Liver fibrosis is a pathological condition characterized by the replacement of normal liver tissue with scar tissue due to a variety of liver diseases, such as hepatitis B, hepatitis C, autoimmune liver diseases, as well as alcohol-induced or nonalcoholic fatty liver disease. It occurs due to a repeated damage and repair process, which is directly related to excessive accumulation of the extracellular matrix [1]. Excessive liver fibrosis can lead to hepatic cirrhosis and loss of liver function as well as digestive tract bleeding and hepatocellular carcinoma [2]. Liver fibrosis is involved in multiple liver cell types, including hepatic parenchymal cells, Kupffer cells, endothelial cells, hepatic stellate cells (HSCs), and infiltrated blood inflammatory cells, which proliferate and replace lost liver cells as well as produce the extracellular matrix in the injured liver [3]. Molecularly, upon the expression of liver disease-induced fibrogenic factors, like transforming growth factor- $\beta$ (TGF- $\beta$ ) as a profibrotic mediator, HSCs will start to proliferate from their quiescent state due to the loss of cytoplasmic retinoid into proliferative and fibrogenic myofibroblasts, which express alpha-smooth muscle actin ( $\alpha$-SMA) and extracellular matrix type I, III, and IV collagen, and depose them into the space of the lost hepatic parenchymal cells, resulting in liver fibrosis [4]. However, the molecular mechanisms of hepatic fibrogenesis remain to be defined, and there is still a lack of effective drugs for the treatment of liver fibrosis [5].

Chinese herbal medicines have demonstrated anti-fibrotic effects in vitro and in vivo [610], The advantage of Chinese herbal formulae (CHFs) is that they use the synergy of various herbs containing different active ingredients to achieve an efficient treatment for human disease although a number of CHFs need to be precisely analyzed for their constituents and active compounds. Treatment of patients with traditional Chinese medicine (TCM) is based on syndrome (ZHENG) differentiation [11]; for example, a sub-type of liver fibrosis is referred to as 
liver depression and spleen deficiency syndrome (LDSDS) in TCM, and it is often treated with Xiaoyaosan (XYS) [12], a classic CHF derived from "Prescriptions of the People's Welfare Pharmacy" in the Song dynasty of China (960-1127 AD). It consists of Bupleurum falcatum L., Angelica archangelica L., Paeonia lactiflora Pall., Atractylodes macrocephala Koidz., Poria cocos (Schw.) Wolf, Glycyrrhiza uralensis Fisch, Zingiber officinale Roscoe and Mentha piperita L. $[12,13]$. Previous studies have shown that XYS treatment alleviates liver injury and improves liver fibrosis in vivo and in vitro [14] through TGFbeta/Smad and Akt/FoxO3 signaling pathways. Molecularly, the formula exerts anxiolytic-like effects through downregulation of TNF- $\alpha$ /JAK2-STAT3 signaling [15]. Since the complexity of CHFs due to their multiple compounds, targets, and biological activities, it has been difficult to assess their real and multiple compounds combined pharmacological effects and mechanisms. Following the recent development of transcriptomics, network pharmacology and systems biology provides novel methodologies for such analyses and a better understanding of the molecular mechanism for different CHFs, like Huangqi decoction [16], Yinchenhao decoction [17], and Fuzhenghuayu formula [18]. They also provide approaches for determining the synergistic effects of active compounds from XYS on liver fibrosis.

In previous study, we have searched five TCM databases (TCM@Taiwan, TCMSP, TCMID, TCM-PTD, and HIT database) to identify the active compounds of XYS and performed bioinformatics analysis of the target genes and signaling pathways. In this study, we developed a novel formula with luteolin, licochalcone A, aloe-emodin, and acacetin (LLAAF) from XYS base on the pharmacology network analysis, and then assessed their anti-liver fibrotic effects in a rat liver fibrosis model induced by $\mathrm{CCl}_{4}$, and predicted the anti-liver fibrotic mechanisms using 
transcriptomics analysis. Furthermore, we verified their anti-liver fibrotic mechanisms using Western blotting experiments in vitro and in vivo.

\section{Materials and methods}

\subsection{Reagents}

Luteolin, licochalcone A, aloe-emodin, acacetin, Paeoniflorin, ferulic Acid and glycyrrhizic Acid all $\geq 98 \%$ pure by HPLC were purchased from Shanghai Traditional Chinese Medicine Standardization Center (Shanghai, China), the chemical structures were showed in Figure S1 and S2C. $\mathrm{CCl}_{4}$ and olive oil were obtained from Sinopharm Chemical Reagent Co., Ltd. (Shanghai, China). The alanine aminotransferase, aspartate aminotransferase, albumin, and total bilirubin kits were purchased from Shino-Test Corporation (Tokyo, Japan). The hyaluronic acid, laminin, type IV collagen, and type III procollagen kits were from Shanghai Enzyme Biotechnology Co., Ltd. (Shanghai, China). Hydroxyproline (Hyp) was purchased from Nanjing Institute of Bioengineering (Nanjing, China), and colchicine was from Banna Pharmaceutical Co., Ltd. (Xishuangbanna, China). Methanol ( $\geq 98 \%$ pure by HPLC) and formic acid ( $\geq 98 \%$ pure by HPLC) were from Thermo Fisher Scientific (Waltham, MA, USA). The antibodies against human smooth muscle actin ( $\alpha$-SMA) and type I collagen A2 (collagen IA2) were from Abcam (Cambridge, MA, USA); the antibodies against p-Akt, Akt, p-Smad3, Smad3, p-STAT3, pFoxO3a, and GAPDH were from Cell Signaling Technology Inc. (Danvers, MA, USA); and the antibodies against c-Myc, FoxO3a, and STAT3 were obtained from Proteintech (Rosemont, IL, USA). Fetal bovine serum (FBS) and Dulbecco's modified Eagle's medium (DMEM) were from Gibco Life Technologies (Gaithersburg, MD, USA), and human TGF- $\beta 1$ was from Promega (Madison, WI, USA). 


\subsection{Preparation of XYS}

The four Chinese herbal medicines of XYS purchased from Shanghai Huanghai Pharmaceutical Co., Ltd. (Shanghai, China), and prepared following the established methods [14], which contained $10 \mathrm{~g}$ of Bupleurum falcatum L., $10 \mathrm{~g}$ of Angelica archangelica L., $10 \mathrm{~g}$ of Paeonia lactiflora Pall., 10 g of Atractylodes macrocephala Koidz., 10 g of Poria cocos (Schw.) Wolf, 5 g of Glycyrrhiza uralensis Fisch, $3 \mathrm{~g}$ of Zingiber officinale Roscoe, and $3 \mathrm{~g}$ of Mentha piperita L. Detection on Paeoniflorin, ferulic acid and glycyrrhizic acid was performed via ultraperformance liquid chromatography-tandem mass spectrometry (UPLC-MS/MS) for the quality control of XYS. (Fig. S2 A-B).

\subsection{Analysis of XYS active compounds and potential gene targets}

The active compounds and potential targets of XYS were retrieved from five different TCM databases (i.e., TCM@Taiwan, TCMSP, TCMID, TCM-PTD, and HIT). We first screened XYS for its pharmacokinetic and pharmacodynamic properties (oral bioavailability, $\mathrm{OB}, \geq 30 \%$ and drug-likeness, DL, $\geq 0.18$ ) for the potential active compounds in these five databases and then performed further network pharmacological analysis. Next, we identified the potential target proteins of XYS through the molecular similarity match tool, such as the simplified molecular input line entry specification (SMILES) in SEA (http://sea.bkslab.org/) and Swiss for target prediction. After that, we expanded the target protein prediction from the STRING database according to the human protein-protein interactions (PPIs) to obtain the liver fibrosis-related targets using the three existing resources, including OMIM, Genecards, and Disgene, with the 
keywords of "hepatic fibrosis" and "liver fibrosis." Finally, we performed Heatmap analysis using the OmicsBean online tool and pathway enrichment analysis using OmicsBean, Reactome, and DAVID (all databases and software information are listed in Supplemental Table 1).

\section{4 mRNA microarray analysis}

This study was approved by the Ethics Committee of Shanghai University of Traditional Chinese Medicine, and all participants provided a written informed consent form before enrollment into this study. Blood samples from chronic hepatitis B patients with or without LDSDS ( $\mathrm{n}=16$ for each group) were collected and subjected to total cellular RNA isolation using the TRIzol reagent (Cat\# 15596-018; Invitrogen, Carlsbad, CA, USA), according to the manufacturer's protocol. The RNA integrity number was assayed using an Agilent Bioanalyzer 2100 (Agilent Technologies, Santa Clara, CA, USA), further purified using an RNeasy Mini Kit (Cat\# 74106; QIAGEN, Hilden, Germany) and an RNase-Free DNase kit (Cat\# 79254; QIAGEN), and then stored at $-80{ }^{\circ} \mathrm{C}$ until use. For the cDNA microarray analysis, each sample was labeled using a Low Input Quick Amp Labeling Kit, One-Color (Cat\# 5190-2305; Agilent Technologies) and hybridized to the cDNA array using a Gene Expression Hybridization Kit (Cat\# 5188-5242; Agilent Technologies), according to the manufacturer's protocol. The arrays were then scanned by using an Agilent Microarray Scanner (Cat\# G2565CA; Agilent Technologies) with default settings, and the raw data were extracted using the Feature Extraction software 10.7 (Agilent Technologies) and normalized using the Quantile algorithm, Gene Spring Software 11.0 (Agilent Technologies). The transcriptomic data of the differentially expressed genes (DEGs) were obtained after the SAS online software analysis for a fold change $>1.5$ and $\mathrm{p}$-value $<0.05$. 
Similarly, liver tissues from the $\mathrm{CCl}_{4}$-treated control and XYS or LLAAF treated rats $(\mathrm{n}=$ 3 for each group) were collected for the isolation of total cellular RNA and cDNA microarray analysis. For the DEGs, we utilized a fold change $>2$ and p-value $<0.05$ as the criteria.

\subsection{Bioinformatics analyses of the DEGs}

We constructed the XYS-induced DEG networks and the XYS- or LLAAF-targeted mRNA networks using Cytoscape software and calculated the related parameters for significant network nodes according to a previous study [19]. We evaluated the network stabilization according to the method of Liu et al. [20]. We then utilized the GeneMAIA tool to construct the PPI network of LLAAF and its four individual compounds and the MCODE tool in Cytoscape software to extract their core subnetwork using $\mathrm{p}<0.05$. After that, we compared the composition of the core subnetwork using the Venn analysis tool and the Omicsbean tool plus the Venn analysis tool to predict the mechanism of action of these different compounds. In addition, we performed gene ontology (GO) and Kyoto Encyclopedia of Genes and Genomes (KEGG) database pathway enrichment analyses using the online Omicsbean analysis tool.

\subsection{Prediction of potential compound combinations of XYS}

We evaluated the relevant network stability parameters, including the network centralization (NC), characteristic path length (CPL), network heterogeneity $(\mathrm{NH})$, and robustness (R) levels using Cytoscape software, according to a previous study [20]. The R levels were calculated using the following formula:

$\boldsymbol{R}=\frac{\boldsymbol{C}}{(\boldsymbol{N}-\mathrm{N} r)}$, where $\mathrm{C}$ is the maximum connected amount, $\mathrm{N}$ is the number of original nodes, and $\mathrm{Nr}$ is the number of knockout nodes. 
We then calculated the network contribution score and the importance of the compound in the network using the entropy method and the weighted summation method. Next, we performed Venn analysis to retain the top $50 \%$ of the screening scores. The network contribution scores were calculated using the following methodologies by converting the actual value of each relevant network stability parameter into an evaluation value:

Positive indicator: $z \boldsymbol{i} \boldsymbol{j}=\frac{x \boldsymbol{i j}-\min (x \boldsymbol{j})}{\max (x \boldsymbol{j})-\min (x \boldsymbol{j})}$

Inverse indicator: $z i j=\frac{\max (x j)-x i j}{\max (x j)-\min (x j)}$

Weight of each indicator: $b i j=\frac{z i j}{\sum n i=1 z i j}, i=1, \ldots, n, j=1, \ldots, m$

Entropy value of each indicator: $e j=-k \sum_{i=1}^{n} b j \ln (b j) ; k=1 / \ln (n) ; k>0 ; e j>0$

Difference coefficient: $\mathbf{d} \boldsymbol{j}=\mathbf{1}-\boldsymbol{e j}$

The difference coefficient was normalized to obtain the weight of each index: $\boldsymbol{w j}=\frac{d j}{\sum_{i=1}^{n} d j}$

The formula for the network contribution score is as follows: Contribution score $=\mathbf{N C} \times$

$W(n c) j+C P L \times W(c p l) j+N H \times W(N H) j+R \times(W(R) j \times 2)$

The high-scoring compounds and combinations were then selected for subsequent experimental verification.

\subsection{Animal experiments}

The animal protocol of this study was approved by the Institutional Animal Care and Use Committee of Shanghai University of Traditional Chinese Medicine (Shanghai, China) and followed the Guidelines for the Care and Use of Laboratory Animals issued by the Chinese 
Council on Animal Research. Specifically, male Wistar rats weighing 150-160 g were purchased from the Shanghai Laboratory Animal Center of the Chinese Academy of Sciences (Shanghai, China) and acclimated to the laboratory conditions for seven days at room temperature (22$26^{\circ} \mathrm{C}$ ) with a relative humidity of $40-70 \%$. The rats were then randomly allocated to the control or the $\mathrm{CCl}_{4}$ treatment group. The rats were intraperitoneally injected with $1 \mathrm{~mL} / \mathrm{kg}$ bodyweight of olive oil or $50 \% \mathrm{CCl}_{4}$ in olive oil at $1 \mathrm{~mL} / \mathrm{kg}$ bodyweight, twice a week for nine weeks. At the end of week 4, the $\mathrm{CCl}_{4}$-treated rats were then randomly divided into three groups, i.e., the control model group, the XYS treatment group, and the LLAAF treatment group. These rats were treated orally with normal saline, XYS extract (method of decocting) at a dose of $6.3 \mathrm{~g} / \mathrm{kg} / \mathrm{d}$, and LLAAF (5.5 mg/kg/d luteolin, $1.5 \mathrm{mg} / \mathrm{kg} / \mathrm{d}$ licochalcone A, $2.5 \mathrm{mg} / \mathrm{kg} / \mathrm{d}$ aloe-emodin, and 2.5 $\mathrm{mg} / \mathrm{kg} / \mathrm{d}$ acacetin) daily for four weeks, respectively. If the animal weight loss reached $20-30 \%$ of that of the control rats, the model rats were euthanized by $\mathrm{CO}_{2}$ and then cervical dislocation. At the end of the experiments, all rats were sacrificed for tissues resection and various analyses.

\subsection{Histopathological analysis of rat liver tissues}

Rat liver tissues were collected, weighed, and sectioned into small pieces. Then, they were fixed in $4 \%$ paraformaldehyde in phosphate buffer and subjected to paraffin embedding for hematoxylin and eosin (H\&E) staining, Sirius Red staining, and Masson's staining, according to a previous study [18]. The Masson-stained tissue sections were quantified by ImageJ software (National Institutes of Health, Bethesda, MD, USA).

\subsection{Cell culture and treatment}


The human HSC lines LX2 and L02 as well as the rat HSC cell line HSC-T6, obtained from the Type Culture Collection of the Chinese Academy of Sciences (Shanghai, China), were maintained in DMEM supplemented with 10\% FBS, penicillin $(100 \mathrm{U} / \mathrm{mL})$, and streptomycin $(100 \mu \mathrm{g} / \mathrm{mL})$ in a humidified incubator with $5 \% \mathrm{CO}_{2}$ at $37{ }^{\circ} \mathrm{C}$, according to a previous study [16]. For cell treatment, HSC-T6 and LX2 cells were cultured in a 6-well plate at a density of 1.0 $\times 10^{6}$ cells/well overnight, and then they were treated with TGF- $\beta 1$ (LX2, $5 \mathrm{ng} / \mathrm{mL}$ and HCS-T6, $20 \mathrm{ng} / \mathrm{mL}$ ) and compound or their combinations, i.e., the compound concentrations were $0.8-3.1$ $\mu \mathrm{M}$ luteolin, 0.8-3.1 $\mu \mathrm{M}$ licochalcone $\mathrm{A}, 3.1-12.5 \mu \mathrm{M}$ aloe-emodin, and 1.5-6.3 $\mu \mathrm{M}$ acacetin, for $24 \mathrm{~h}$.

\subsection{Cell viability assay}

Cells were seeded into 96 -well plates at a density of $5 \times 10^{3}$ cells/well, grown overnight, and then treated with the above-named compounds and their combinations for $24 \mathrm{~h}$. At the end of each experiment, $20 \mu \mathrm{L}$ of the tetrazolium compound 3-(4,5-dimethylthiazol-2-yl)-5-(3carboxymethoxyphenyl)-2-(4-sulfophenyl)-2H-tetrazolium (MTT) (Thermo, Waltham, MA USA) was added to the cell culture, the cells were incubated for an additional $3.5 \mathrm{~h}$, and the absorbance rates were measured using a Synergy2 spectrophotometer (BioTek, Winooski, VT, USA) at $490 \mathrm{~nm}$. The experiment was performed in triplicate and repeated at least three times.

\subsection{Western blot}

Liver tissue specimens were frozen in liquid nitrogen, ground, lysed, sonicated for complete dissolution, and then centrifuged at $12,000 \mathrm{rpm}$ and $4{ }^{\circ} \mathrm{C}$ for $30 \mathrm{~min}$. Protein samples of $100 \mu \mathrm{g}$ each were separated by sodium dodecyl sulfate-polyacrylamide gel electrophoresis and 
transferred onto nitrocellulose membranes (Millipore, Billerica, MA, USA). For western blotting, the membrane was incubated with a blocking buffer containing $5 \%$ nonfat dry milk in Tris-based saline-Tween 20 (TBS-T) at room temperature for $1 \mathrm{~h}$ and then with polyclonal primary antibodies against $\alpha$-SMA, collagen IA2, and GAPDH, respectively, at $4{ }^{\circ} \mathrm{C}$ overnight. On the next day, the membranes were washed with TBS-T three times for 10 min each and then incubated with an infrared dye-conjugated secondary antibody from Cell Signaling Technology at room temperature for $1 \mathrm{~h}$. The protein bands were quantified using an Odyssey Infrared Imager (LI-COR Biosciences, Lincoln, NE, USA), according to a previous study [20].

\subsection{Statistical analysis}

The data were expressed as the mean \pm standard deviation for the one-way analysis of variance and rank-sum tests using SPSS 18.0 software (SPSS, Chicago, IL, USA). A $p$ value $<0.05$ was considered statistically significant.

\section{Results}

\subsection{Identification of active compounds, targets and signaling pathways of XYS}

In this study, we first screened and found a total of 174 active compounds in XYS from the five TCM databases using our pharmacokinetics and pharmacodynamics screening criteria $(\mathrm{OB} \geq$ $30 \%$ and $\mathrm{DL} \geq 0.18$ ). We then obtained 258 potential target proteins from these five databases and 309 and 525 predicted target proteins, respectively, after performing the SwissDock and SEA structural similarity prediction analyses. The PPI network analysis showed 601 related target proteins in the network using a PPI score $>0.850$, compared to 258 target proteins in the PPI network. Next, we searched the OMIM, DisgeneT, and GeneCard databases using the keywords 
of "hepatic fibrosis" and "liver fibrosis" and found a total of 1191 liver fibrosis-related target genes, while 1116 DEGs with LDSDS treatment were identified from the mRNA microarray data. The microarray data of blood samples from chronic hepatitis B patients with or without LDSDS and from healthy volunteers were overlapped, and the DEGs of the XYS, LDSDS, and liver fibrosis groups identified 181 targets (Fig. 1A). Compared with the XYS active compoundrelated target proteins, the data showed 14 target proteins directly related to XYS. The GO and KEGG pathway enrichment analyses showed that these 14 XYS target genes formed the PI3KAkt-FoxO, AMPK, Hippo, and Jak-STAT pathways (Fig. 1C-D). The heatmap of these targets between chronic hepatitis B patients with or without LDSDS were shown in Fig. 1B.

Next, we constructed the PPI network for the XYS-LDSDS-liver fibrosis progression. The nodes of the PPI network included 165 compounds, 14 proteins directly targeted by XYS from the TCM database, 166 proteins indirectly targeted by XYS from structural similarity prediction, and 184 intermediately targeted proteins. The core network of XYS-LDSDS-liver fibrosis progression was obtained using the five most important topological parameters, including degree, betweenness of centrality, closeness of centrality, clustering coefficient, and topological coefficient analysis. The nodes of the PPI network included six Chinese herbal medicines and 17 compounds, and XYS was directly associated with nine target proteins; meanwhile, XYS was predicted to target 6 proteins directly, 30 indirectly, and 102 intermediately as well as 15 signaling pathways (Fig. 1E).

\subsection{Evaluation of compounds in the XYS-LDSDS-liver fibrosis core network}

To identify the core compounds of the XYS-LDSDS-liver fibrosis network, we knocked out the related target proteins of 17 compounds and utilized the entropy method as well as the weighted 
summation method to generate the network contribution scores by integrating the NC, CPL, NH, and R parameters, which are directly related to the stability of the networks, using Cytoscape 3.2.1 software (Figure 2). After that, we selected eight compounds from the network, i.e., luteolin, lupiwighteone, beta-sitosterol, stigmasterol, licochalcone A, aloe-emodin, eriodictyol, and acacetin. We then knocked out the related targets using one or more of these eight compounds and obtained 255 different combinations based on the network contribution score analysis for their feasibility ranking; the top 10 compound combinations and their scores are listed in Table 1.

\subsection{Effects of XYS compounds and their combinations on regulating cell viability in vitro}

Based on the network analyses, we selected the top six compound combinations to assay their effects on regulating cell viability. First, we detected six single compound effect on the cell viability on LX2 cells. While using the minimum effective dose of these individual six compounds to combine 4 different combinations, it was showed that the that the best effective combination was the luteolin, licochalcone, aloe-emodin, acacetin formula (LLAAF; Fig. 3A). And similar finding were confirmed in another mouse HSC-T6 cells (Fig.3B)

\subsection{Effect of LLAAF on $\alpha$-SMA and collagen I expressions in vitro}

Based on the effective doses of the individual compounds in LLAAF on reducing the LX2 cell viability, we selected the half-doses of these compounds as the highest dose and the second and third half-dilutions as the medium and low doses, respectively, i.e., $3.1 \mu \mathrm{M}$ luteolin $+3.1 \mu \mathrm{M}$ licochalcone $\mathrm{A}+12.5 \mu \mathrm{M}$ aloe-emodin $+6.3 \mu \mathrm{M}$ acacetin; $1.6 \mu \mathrm{M}$ luteolin $+1.6 \mu \mathrm{M}$ licochalcone $\mathrm{A}+6.3 \mu \mathrm{M}$ aloe-emodin $+3.1 \mu \mathrm{M}$ acacetin; $0.8 \mu \mathrm{M}$ luteolin $+0.8 \mu \mathrm{M}$ licochalcone 
$\mathrm{A}+3.1 \mu \mathrm{M}$ aloe-emodin $+1.6 \mu \mathrm{M}$ acacetin. We stimulated LX2 and HSC-T6 cells with TGF- $\beta 1$ and found that the levels of $\alpha$-SMA and collagen I were significantly increased; however, the high and medium doses of LLAAF were able to dramatically reduce the expression of $\alpha$-SMA and collagen I in these cells $(P<0.05)$, but the low-dose LLAAF treatment only reduced the expression levels of $\alpha$-SMA and collagen I in HSC-T6 cells ( $P<0.05$; Fig. 4 A and C). Of note, any single compound at the same concentration did not show any effects on changing the $\alpha$-SMA and collagen I expression in these cells (Fig. 4 B and D).

\subsection{Effects of XYS and LLAAF on liver fibrosis in vivo}

Next, we assessed the effects of LLAAF and XYS on reducing liver fibrosis in the $\mathrm{CCl}_{4}$ model of Wistar rats. We found that XYS was able to improve the rat liver weight index, pathological index, serum liver function index, serum liver fiber index, and liver tissue fibers in vivo[14]. The histopathological data showed establishment of $\mathrm{CCl}_{4}$-induced liver fibrosis in rats treated twice a week for nine weeks (Fig. 5), whereas treatment of rats with either LLAAF or XYS significantly attenuated the $\mathrm{CCl}_{4}$-induced liver fibrosis. Specifically, $\mathrm{H} \& \mathrm{E}$ staining showed lower levels of edema and inflammation of liver cells in the XYS and LLAAF groups than in the $\mathrm{CCl}_{4}$ controls (Fig. 5A), whereas Masson's staining showed that fibrosis and paraplastic connective tissues were built up in the $\mathrm{CCl}_{4}$ rats vs. mild fibrosis in the XYS and LLAAF rats (Fig. 5B). This semiquantitative analysis indicates reduced levels of collagen fibers in the XYS-M- and LLAAFtreated rats compared to the model rats $(P<0.01 ;$ Fig. 5C). Furthermore, western blot analysis revealed that the levels of $\alpha$-SMA and collagen I as well as the Hyp content in the $\mathrm{CCl}_{4}$-induced rat liver tissue was higher than that in the XYS and LLAAF-treated rats $(P<0.05$; Fig. 5D-E). In 
addition, treatment with LLAAF showed an even better reduction of the Hyp content and $\alpha$-SMA level in the liver tissues.

\subsection{Prediction of anti-liver fibrotic mechanisms of XYS and LLAAF}

To explore the underlying mechanisms of XYS and LLAAF anti-fibrotic efficacy, we collected liver tissue samples from the $\mathrm{CCl}_{4}$ model, XYS-treated rats, and LLAAF-treated rats for cDNA and lncRNA microarray analyses. And the total DEGs of XYS and LLAAF compared with $\mathrm{CCl}_{4}$ model group were analyzed (Fig. 6A and 6B). The corresponding KEGG pathways were asloanalyzed individually. (Fig. 6C and D) When compared the two KEGG pathways, it was showed that the XYS treatment was mainly related to p53, cell cycle, viral carcinogenesis, JakSTAT3, AMPK, and FoxO signaling, while the LLAAF treatment was mainly associated with PI3K-Akt, AMPK, FoxO, Jak-STAT3, P53, cell cycle, focal adhesion, and PPAR signaling (Fig. $6 \mathrm{E})$.

\subsection{LLAAF regulates Jak-STAT and PI3K-Akt-FoxO signaling}

These bioinformatics analysis results were performed in vitro and in vivo. The LX2 cells were treated with TGF- $\beta 1$, an inducer of liver fibrosis, and found that such a treatment significantly increased the levels of p-FoxO3a, p-STAT3, p-Akt, p-Smad3, and c-Myc in LX2 cells $(P<0.05)$; whereas different doses of LLAAF treatment significantly downregulated their expression $(P<$ 0.05; Fig. 7A). And we also detected the Jak-STAT and PI3K-Akt-FoxO signaling in the liver samples from animal model, found the LLAAF significantly downregulated the Jak-STAT and PI3K-Akt-FoxO signaling Jak-STAT and PI3K-Akt-FoxO signaling similar with the XYS.

(Fig.7B) 


\section{Discussion}

Liver fibrosis is a liver wound healing process which response to different types of liver diseaseinduced hepatocyte injury. It reflects an imbalance between fibrosis progression and regression [5] and confers a significant risk for morbidity and mortality [21]. The advanced stage of liver cirrhosis is only cured by a liver transplantation [2]; thus, novel treatment options and a better understanding of the underlying pathogenesis are critical to cure and improve the quality of life of these patients. In previous study, we evaluate a Chinese herbal formula named XYS, the antiliver fibrotic activity in vitro and in vivo[22]. Network pharmacology combined with transcriptomics analysis was used to identify active compounds and gene targets of XYS. 174 active compounds in XYS and participation of the PI3K-Akt-FoxO, AMPK, and Hippo and JakSTAT pathways. Indeed, XYS contains more than 174 active compounds, making it difficult to assess the ones responsible for controlling liver fibrosis in vivo and in vivo.

In the current study, By analyzing the topology parameters according to previous studies $[19,23$, 24], we constructed the XYS-LDSDS-liver fibrosis core networks, based on the network contribution score analysis for their feasibility ranking. We utilized four parameters, i.e., NC, $\mathrm{CPL}, \mathrm{NH}$, and $\mathrm{R}$, to relate the stability of the bioinformatics networks according to a previous study [25]. The method of entropy weighting is a common way to integrate multiple indicators [26]; the greater the entropy of an indicator, the greater the impact of the indicator on the comprehensive evaluation. After evaluating the network contribution scores, we identified eight core compounds and the top 10 combinations with anti-fibrotic activities. In the cell based assay, the combination of luteolin, licochalcone A, aloe-emodin and acacetin were generated as the most effective combination from XYS. 
Specifically, luteolin is in a variety of dietary sources, such as celery, broccoli, green pepper, and parsley [27, 28]. In addition, luteolin can attenuate the inflammatory responses in dendritic cells, indicating its therapeutic potential against ulcerative colitis [29], and protects against $\mathrm{CCl}_{4}$-induced hepatotoxicity in combination with metformin [30]. Moreover, licochalcone A, a chalconoid, can be found in the roots of Glycyrrhiza glabra or Glycyrrhiza inflata [31-34]. It possesses antiproliferative and proapoptotic activities by reactive oxygen species-mediated cell cycle arrest and apoptosis in human bladder cancer cells [35] and inhibits proliferation, migration, and invasion of oral squamous cell carcinoma cells by regulating the PI3K/AKT signaling pathway [36]. Similarly, aloe-emodin, which is isolated from aloe and Rheum palmatum, has antiproliferative effects and induces cell apoptosis [37, 38]. It also inhibits monolayer growth and anchorage-independent growth of androgen refractory prostate cancer PC3 cells by targeting the mTOR complex II [39]. More importantly, aloe-emodin has shown potential to treat hepatitis B viral infection [40], a condition that induces liver fibrosis, and to alleviate cardiac fibrosis via suppression of cardiac fibroblast activation by metastasis-associated protein 3 upregulation [41]. Functionally, acacetin has been reported to regulate the reciprocal differentiation of $\mathrm{T}$ helper 17 and regulatory $\mathrm{T}$ cells as well as the symptoms of collagen-induced arthritis in mice [42]. Additionally, it protects cardiomyocytes against hypoxia/reoxygenation injury by activating a series of intracellular signals involved in antioxidation, anti-inflammation, and antiapoptosis [43]. Taken together, these four compounds all possess anti-inflammatory, antioxidative, antiproliferative, antibacterial, and antiviral activities through multiple gene pathways. Therefore, their combination could yield an even greater anti-liver fibrotic activity in vitro and in vivo, as demonstrated in the current study. 
The anti-fibrotic effect of XYS and LLAAF were classified in cells as well as in the $\mathrm{CCl}_{4}$-induced liver fibrosis rats. HSCs are the most important cell type involved in liver fibrosis and the major producer of the extracellular matrix during liver injury $[44,45]$. Thus, the current study utilized human and rat HSCs to assess the anti-liver fibrotic activity of the top four compound combinations. These data indicated that the combination of LLAAF was the most effective for cell viability and suppression of $\alpha$-SMA and collagen I expression in vitro. In the $\mathrm{CCl}_{4}$-induced liver fibrosis rat model, both XYS and LLAAF were able to reduce cell damage and steatosis, the Hyp content, and the levels of $\alpha$-SMA and collagen I in liver tissues, suggesting that LLAAF could be able to alleviate liver fibrosis. We performed cDNA microarray analysis and then analyzed the PPI network of the XYS-targeting proteins and the LLAAF-targeting proteins. According to the network topology characteristics and the pathway enrichment analysis, we found that the Jak-STAT and PI3K-Akt-FoxO signaling pathways were the main pathways involved; while previous studies have revealed that Jak-STAT signaling is involved in liver fibrosis and that activation of the Jak-STAT signaling pathway can promote the development and progression of liver fibrosis $[46,47]$. Other studies have shown that STAT3 phosphorylation occurs during liver fibrosis [48] and that PI3K-Akt-FoxO signaling is involved in chronic liver disease and liver fibrosis [49]. Again, Akt activation leads to FoxO phosphorylation and departure from the cell nucleus, and reduced FoxO expression also results in the development of liver fibrosis. Besides, we also assessed another classic target, Smad3 [50], which is highly relevant to liver fibrosis and acts as a signaling mediator for the TGF- $\beta$ family for cell proliferation, differentiation, and death [50]. In addition, blockage of Smad3 phosphorylation has been demonstrated to prevent liver fibrosis [51]. In this regard, the current study confirmed the p-STAT3/STAT3, p-Smad3/Smad3, p-Akt/Akt, and p-FoxO3a/FoxO3a ratios as well as c-Myc 
expression in vitro and in vivo. The XYS modified PPI networks were mainly related to p53, cell cycle, viral carcinogenesis, Jak-STAT3, AMPK, and FoxO signaling; while the LLAAF-modified PPI networks were associated with PI3K-Akt, AMPK, FoxO, Jak-STAT3, P53, cell cycle, focal adhesion, and PPAR signaling.

\section{Conclusions}

In summary, the current study developed a novel formula, LLAAF with luteolin, licochalcone A, aloe-emodin and acacetin derived from XYS, and demonstrated the anti-fibrotic effects of XYS and LLAAF. The anti-liver fibrotic mechanism of LLAAF may be related to the downregulation of Jak-STAT and PI3K-Akt-FoxO activity, indicating that LLAAF should be further evaluated as a novel therapeutic formula to control liver fibrosis.

\section{List of Abbreviations:}

XYS, Xiaoyaosan decoction; LLAAF, luteolin, licochalcone A, aloe-emodin, and acacetin formula; TCM, Traditional Chinese Medicine; CC14, carbon tetrachloride; EGGs, Different expressed genes; KEGG, Kyoto Encyclopedia of Genes and Genomes; GO, Gene Ontology; FoxO, forkhead box transcription factors class O; TGF $\beta$, Transforming Growth Factor Beta ; AMPK, AMP-activated protein kinase; MAPK, mitogen-activated protein kinase ; PPAR, Peroxisome proliferator-activated receptor; ECM, extracellular matrix; HSCs, hepatic stellate cells; $\alpha$-SMA, smooth muscle $\alpha$-actin; ALT, alanine aminotransferase; AST, aspartate aminotransferase; ALB, albumin; TBIL, total hyaluronic acid (HA); LN, laminin; IV-C, type IV collagen; PC-III, type III procollagen; H\&E, haematoxylin and eosin; Hyp, hydroxyproline; AOD, average optical density; LC-MS, Liquid chromatography-mass spectrometry; 


\section{Declarations:}

\section{Ethics approval and consent to participate:}

This study was approved by the Ethics Committee of Shanghai University of Traditional Chinese

Medicine, and all participants provided a written informed consent form before enrollment into this study.

\section{Consent for publication}

Not applicable.

\section{Availability of data and materials}

The datasets used and analysed during the current study are available from the corresponding author on reasonable request.

\section{Competing interests}

The authors declare that they have no competing interests.

\section{Funding.}

This work was supported by grants from Major Project of Shanghai Municipal S\&T Commission (No 19401972300) and National Science Foundation of China (81330084). 


\section{Author Contributions}

Mingyu Sun and Shi-Bing Su conceptualize the study; Yuan Zhou, Rong Wu, Fei-Fei Cai and Wen-Jun Zhou completed the experiments; Yuan Zhou, Rong Wu and Qi-Long Chen analyzed the data; Y Zhou and R Wu wrote the manuscript; Yi-Yu Lu, Mingyu Sun and SB Su revised the manuscript; All authors read and approved the final manuscript.

\section{Acknowledgements}

Not applicable.

\section{References}

1. Seki E, Schwabe RF: Hepatic Inflammation and Fibrosis: Functional Links and Key Pathways. Hepatology 2015, 61(3):1066-1079.

2. Koyama Y, Brenner DA: New therapies for hepatic fibrosis. Clinics and Research in Hepatology and Gastroenterology 2015, 39:S75-S79.

3. Higashi T, Friedman SL, Hoshida Y: Hepatic stellate cells as key target in liver fibrosis. Advanced Drug Delivery Reviews 2017, 121:27-42.

4. Tsuchida T, Friedman SL: Mechanisms of hepatic stellate cell activation. Nature Reviews Gastroenterology \& Hepatology 2017, 14(7):397-411.

5. Yoon YJ, Friedman SL, Lee YA: Antifibrotic Therapies: Where Are We Now? Seminars in Liver Disease 2016, 36(1):87-98.

6. Liu C, Hu Y, Xu L, Liu C, Liu P: Effect of Fuzheng Huayu formula and its actions against liver fibrosis. Chin Med 2009, 4:12.

7. Dong S, Su SB: Advances in mesenchymal stem cells combined with traditional Chinese medicine therapy for liver fibrosis. J Integr Med 2014, 12(3):147-155.

8. Zhang L, Schuppan D: Traditional Chinese Medicine (TCM) for fibrotic liver disease: hope and hype. Journal of hepatology 2014, 61(1):166-168.

9. Sanodiya BS, Thakur GS, Baghel RK, Prasad GB, Bisen PS: Ganoderma lucidum: a potent pharmacological macrofungus. Curr Pharm Biotechno/2009, 10(8):717-742. 
10. Tang Y, Liao Y, Kawaguchi-Sakita N, Raut V, Fakhrejahani E, Qian N, Toi M: Sinisan, a traditional Chinese medicine, attenuates experimental chronic pancreatitis induced by trinitrobenzene sulfonic acid in rats. J Hepatobiliary Pancreat Sci 2011, 18(4):551-558.

11. Su SB, Jia W, Lu A, Li S: Evidence-Based ZHENG: A Traditional Chinese Medicine Syndrome 2013. Evid Based Complement Alternat Med 2014, 2014:484201.

12. Liver Disease Committee CAoIM: [Guidelines for the diagnosis and treatment of liver fibrosis with integrated traditional Chinese and Western medicine (2019 edition)]. Zhonghua gan zang bing za zhi = Zhonghua ganzangbing zazhi = Chinese journal of hepatology 2019, 27(7):494-504.

13. Tian JS, Peng GJ, Wu YF, Zhou JJ, Xiang H, Gao XX, Zhou YZ, Qin XM, Du GH: A GC-MS urinary quantitative metabolomics analysis in depressed patients treated with TCM formula of Xiaoyaosan. J Chromatogr B Analyt Technol Biomed Life Sci 2016, 1026:227-235.

14. Zhou Y, Wu R, Cai FF, Zhou WJ, Lu YY, Zhang H, Chen QL, Su SB: Xiaoyaosan decoction alleviated rat liver fibrosis via the TGFbeta/Smad and Akt/FoxO3 signaling pathways based on network pharmacology and transcriptomic analysis. J Ethnopharmaco/2020:113021.

15. Li XJ, Ma QY, Jiang YM, Bai XH, Yan ZY, Liu Q, Pan QX, Liu YY, Chen JX: Xiaoyaosan exerts anxiolytic-like effects by down-regulating the TNF-alpha/JAK2-STAT3 pathway in the rat hippocampus. Sci Rep 2017, 7(1):353.

16. Song YN, Zhang GB, Lu YY, Chen QL, Yang L, Wang ZT, Liu P, Su SB: Huangqi decoction alleviates dimethylnitrosamine-induced liver fibrosis: An analysis of bile acids metabolic mechanism. J Ethnopharmaco/2016, 189:148-156.

17. Cai FF, Bian YQ, Wu R, Sun Y, Chen XL, Yang MD, Zhang QR, Hu Y, Sun MY, Su SB: Yinchenhao decoction suppresses rat liver fibrosis involved in an apoptosis regulation mechanism based on network pharmacology and transcriptomic analysis. Biomed Pharmacother 2019, 114:108863.

18. Dong S, Cai FF, Chen QL, Song YN, Sun Y, Wei B, Li XY, Hu YY, Liu P, Su SB: Chinese herbal formula Fuzheng Huayu alleviates $\mathrm{CCl}$-induced liver fibrosis in rats: a transcriptomic and proteomic analysis. Acta Pharmacol Sin 2018, 39(6):930-941.

19. Shi SH, Cai YP, Cai XJ, Zheng XY, Cao DS, Ye FQ, Xiang Z: A network pharmacology approach to understanding the mechanisms of action of traditional medicine: Bushenhuoxue formula for treatment of chronic kidney disease. PLoS One 2014, 9(3):e89123.

20. Liu Y, Wang M, Luo Y, Chen C, Lu Y, Shi Y, Tang C, Zhou Q, Zhang H, Hu Y et al: MiRNA-target network analysis identifies potential biomarkers for Traditional Chinese Medicine (TCM) syndrome development evaluation in hepatitis B caused liver cirrhosis. Sci Rep 2017, 7(1):11054.

21. Hernandez-Gea V, Friedman SL: Pathogenesis of Liver Fibrosis. In: Annual Review of Pathology: Mechanisms of Disease, Vol 6. Edited by Abbas AK, Galli SJ, Howley PM, vol. 6; 2011: 425-456. 
22. Zhou Y, Wu R, Cai F-F, Zhou W-J, Lu Y-Y, Zhang H, Chen Q-L, Su S-B: Xiaoyaosan decoction alleviated rat liver fibrosis via the TGF $\beta /$ Smad and Akt/FoxO3 signaling pathways based on network pharmacology analysis. J Ethnopharmaco/2021, 264:113021.

23. Wieczorek J, Malik-Sheriff RS, Fermin Y, Grecco HE, Zamir E, Ickstadt K: Uncovering distinct protein-network topologies in heterogeneous cell populations. BMC Syst Biol 2015, 9:24.

24. Han Y, Li H, Lang Y, Zhao Y, Sun H, Zhang P, Ma X, Han J, Wang Q, Zhou J et al: The Effects of Acute GABA Treatment on the Functional Connectivity and Network Topology of Cortical Cultures. Neurochem Res 2017, 42(5):1394-1402.

25. Eloundou-Mbebi JM, Kuken A, Omranian N, Kleessen S, Neigenfind J, Basler G, Nikoloski Z: A network property necessary for concentration robustness. Nat Commun 2016, 7:13255.

26. Harvey RA, Hayden JD, Kamble PS, Bouchard JR, Huang JC: A comparison of entropy balance and probability weighting methods to generalize observational cohorts to a population: a simulation and empirical example. Pharmacoepidemiol Drug Saf2017, 26(4):368-377.

27. Shimoi K, Okada H, Furugori M, Goda T, Takase S, Suzuki M, Hara Y, Yamamoto H, Kinae N: Intestinal absorption of luteolin and luteolin 7-O- $\beta$-glucoside in rats and humans. FEBS Letters 1998, 438(3):220-224.

28. Lopez-Lazaro M: Distribution and biological activities of the flavonoid luteolin. Mini reviews in medicinal chemistry 2009, 9(1):31-59.

29. Kim WS, Song HY, Han JM, Byun EB: GLM, a novel luteolin derivative, attenuates inflammatory responses in dendritic cells: Therapeutic potential against ulcerative colitis. Biochemical and biophysical research communications 2019.

30. Yan Y, Jun C, Lu Y, Jiangmei S: Combination of metformin and luteolin synergistically protects carbon tetrachloride-induced hepatotoxicity: Mechanism involves antioxidant, anti-inflammatory, antiapoptotic, and Nrf2/HO-1 signaling pathway. BioFactors (Oxford, England) 2019, 45(4):598606.

31. Fu Y, Hsieh TC, Guo J, Kunicki J, Lee MY, Darzynkiewicz Z, Wu JM: Licochalcone-A, a novel flavonoid isolated from licorice root (Glycyrrhiza glabra), causes $\mathrm{G} 2$ and late-G1 arrests in androgen-independent PC-3 prostate cancer cells. Biochemical and biophysical research communications 2004, 322(1):263-270.

32. Friis-Moller A, Chen M, Fuursted K, Christensen SB, Kharazmi A: In vitro antimycobacterial and antilegionella activity of licochalcone A from Chinese licorice roots. Planta medica 2002, 68(5):416-419.

33. Chen M, Theander TG, Christensen SB, Hviid L, Zhai L, Kharazmi A: Licochalcone A, a new antimalarial agent, inhibits in vitro growth of the human malaria parasite Plasmodium falciparum and protects mice from P. yoelii infection. Antimicrobial agents and chemotherapy 1994, 38(7):1470-1475. 
34. Dao TT, Nguyen PH, Lee HS, Kim E, Park J, Lim SI, Oh WK: Chalcones as novel influenza A (H1N1) neuraminidase inhibitors from Glycyrrhiza inflata. Bioorganic \& medicinal chemistry letters 2011, 21(1):294-298.

35. Hong SH, Cha HJ, Hwang-Bo H, Kim MY, Kim SY, Ji SY, Cheong J, Park C, Lee H, Kim GY et al: Anti-Proliferative and Pro-Apoptotic Effects of Licochalcone A through ROS-Mediated Cell Cycle Arrest and Apoptosis in Human Bladder Cancer Cells. Int J Mol Sci 2019, 20(15).

36. Hao $\mathrm{Y}$, Zhang $\mathrm{C}$, Sun $\mathrm{Y}, \mathrm{Xu} \mathrm{H}$ : Licochalcone A inhibits cell proliferation, migration, and invasion through regulating the PI3KJAKT signaling pathway in oral squamous cell carcinoma.

OncoTargets and therapy 2019, 12:4427-4435.

37. Guo J, Xiao B, Zhang S, Liu D, Liao Y, Sun Q: Growth inhibitory effects of gastric cancer cells with an increase in $\mathrm{S}$ phase and alkaline phosphatase activity repression by aloe-emodin. Cancer biology \& therapy 2007, 6(1):85-88.

38. Acevedo-Duncan M, Russell C, Patel S, Patel R: Aloe-emodin modulates PKC isozymes, inhibits proliferation, and induces apoptosis in U-373MG glioma cells. International immunopharmacology 2004, 4(14):1775-1784.

39. Liu K, Park C, Li S, Lee KW, Liu H, He L, Soung NK, Ahn JS, Bode AM, Dong Z et al: Aloeemodin suppresses prostate cancer by targeting the mTOR complex 2. Carcinogenesis 2012, 33(7):1406-1411.

40. Parvez MK, Al-Dosari MS, Alam P, Rehman M, Alajmi MF, Alqahtani AS: The anti-hepatitis B virus therapeutic potential of anthraquinones derived from Aloe vera. Phytotherapy Research, $0(0)$.

41. Xiao D, Zhang Y, Wang R, Fu Y, Zhou T, Diao H, Wang Z, Lin Y, Li Z, Wen L et al: Emodin alleviates cardiac fibrosis by suppressing activation of cardiac fibroblasts via upregulating metastasis associated protein 3. Acta Pharmaceutica Sinica B 2019, 9(4):724-733.

42. Liu L, Yang J, Zu B, Wang J, Sheng K, Zhao L, Xu W: Acacetin regulated the reciprocal differentiation of Th17 cells and Treg cells and mitigated the symptoms of collagen-induced arthritis in mice. Scandinavian journal of immunology 2018, 88(4):e12712.

43. Wu WY, Li YD, Cui YK, Wu C, Hong YX, Li G, Wu Y, Jie LJ, Wang Y, Li GR: The Natural Flavone Acacetin Confers Cardiomyocyte Protection Against Hypoxia/Reoxygenation Injury via AMPKMediated Activation of Nrf2 Signaling Pathway. Front Pharmacol2018, 9:497.

44. Geerts A: History, heterogeneity, developmental biology, and functions of quiescent hepatic stellate cells. Semin Liver Dis 2001, 21(3):311-335.

45. Eng FJ, Friedman SL: Fibrogenesis I. New insights into hepatic stellate cell activation: the simple becomes complex. Am J Physiol Gastrointest Liver Physio/2000, 279(1):G7-G11.

46. Tang LY, Heller M, Meng Z, Yu LR, Tang Y, Zhou M, Zhang YE: Transforming Growth Factorbeta (TGF-beta) Directly Activates the JAK1-STAT3 Axis to Induce Hepatic Fibrosis in Coordination with the SMAD Pathway. J Biol Chem 2017, 292(10):4302-4312. 
47. Kong X, Horiguchi N, Mori M, Gao B: Cytokines and STATs in Liver Fibrosis. Front Physio/ 2012, 3:69.

48. Lakner AM, Moore CC, Gulledge AA, Schrum LW: Daily genetic profiling indicates JAKISTAT signaling promotes early hepatic stellate cell transdifferentiation. World J Gastroentero/ 2010 , 16(40):5047-5056.

49. Park SJ, Sohn HY, Yoon J, Park SI: Down-regulation of FoxO-dependent c-FLIP expression mediates TRAIL-induced apoptosis in activated hepatic stellate cells. Cell Signa/2009, 21(10):1495-1503.

50. Massague J: TGF-beta signal transduction. Annu Rev Biochem 1998, 67:753-791.

51. Hernandez-Aquino E, Zarco N, Casas-Grajales S, Ramos-Tovar E, Flores-Beltran RE, Arauz J, Shibayama M, Favari L, Tsutsumi V, Segovia J et al. Naringenin prevents experimental liver fibrosis by blocking TGFbeta-Smad3 and JNK-Smad3 pathways. World J Gastroenterol 2017, 23(24):4354-4368. 


\section{Figure Legends}

Figure 1. Identification of the active compounds, gene targets, and signaling pathways of XYS. (A) mRNA microarray analysis of differentially expressed genes (DEGs) in the XYS, LDSDS, and liver fibrosis groups. (B) Heatmap of DEGs between chronic hepatitis B patients with or without LDSDS ( $n=16$ in each group). (C) The GO terms of the pathway analysis directly related to XYS. (D) The XYS-LDSDS-liver fibrosis core network analysis. Green, herbs; orange, compounds; indigo, target proteins directly related to XYS; purple, predicted target proteins directly related to XYS; dark blue, target proteins indirectly related to XYS; light blue, target proteins intermediately related to XYS; dark green, predicted target proteins intermediately related to XYS; deep red, core pathway; pink, noncore pathway. (E) The KEGG enrichment analysis of the XYS-LDSDS-liver fibrosis core network.

Figure 2. Contribution score analysis of the XYS-LDSDS-liver fibrosis core network. (A)

Network centralization (NC), characteristic path length (CPL), network heterogeneity (NH), and robustness (R) scores of the 17 compounds by using the entropy method and the weighted summation method, which were directly related to the network stability using Cytoscape 3.2.1 software. (B) Analysis of the contribution scores of the 17 compounds by combinations of the NC, CPL, NH and R parameters.

Figure 3. Effects of six individual XYS compounds and their combinations on regulating cell viability. (A) Cell viability assay. Four top compound combination from network analysis show the effect on the LX2 cells. (B) Cell viability assay. Four top compound combination from 
network analysis show the effect on the HSC-T6 cells. . ${ }^{*} P<0.05$ and ${ }^{* *} P<0.01$, compared to the control cells. (C) The most active compounds combination in the LX2 and HXC-T6 cells.

Figure 4. Effect of LLAAF on regulating protein expression in vitro. (A) Western blot. LX2 cells were grown and treated with a high, medium, or low dose of LLAAF and then subjected to western blot analysis of $\alpha$-SMA and collagen I expression. Quantified data of the western blots

are shown in the corresponding graphs. (B) Western blot. LX2 cells were grown and treated with a medium dose of LLAAF and its compounds and then subjected to western blot analysis of $\alpha$ SMA and collagen I expression. (C) Western blot. HSC-T6 cells were grown and treated with a high, medium, or low dose of LLAAF and then subjected to western blot analysis of $\alpha$-SMA and collagen I expression. (D) Western blot. HSC-T6 cells were grown and treated with a medium dose of LLAAF and its compounds and then subjected to western blot analysis of $\alpha$-SMA and collagen I expression. Quantified data of the western blots are shown in the corresponding graphs. $\# P<0.05$ and $\# \# P<0.01$, compared to the $\mathrm{CCl}_{4}$-control group; $* P<0.05$ and $* * P<$ 0.01, compared to the model group.

Figure 5. Effects of XYS-M and LLAAF treatment on reduction of rat liver fibrosis. (A) H\&E staining (200× magnification). (B) Masson's staining (100×). (C) Semi-quantitative analysis of the collagen fibers. (D) The hydroxyproline (Hyp) content. (E) Western blot. The levels of alpha-smooth muscle actin ( $\alpha$-SMA) and collagen I were analyzed using western blot of rat liver fibrotic tissues. \#\#P<0.01, compared to the control group; $* P<0.05, * * P<0.01$, compared to the model group. 
Figure 6. Bioinformatics analysis of the XYS- and LLAAF-modified PPI networks. (A)

XYS network. (B) LLAAF network. (C) KEGG enrichment analysis of the XYS network. (D) KEGG enrichment analysis of the LLAAF network. (E) Pathway analysis between XYS and LLAAF.

Figure 7. Effect of LLAAF treatment on regulation of Jak-STAT and PI3K-Akt-FoxO signaling. (A) Western blot. LX2 cells were grown, treated with TGF- $\beta 1$ and then with LLAAF for $24 \mathrm{~h}$, and then subjected to western blot analysis of protein expression. (B) Western blot. Liver tissues were resected from rats and subjected to western blot analysis of alpha-smooth muscle actin ( $\alpha$-SMA) and collagen I expression. Quantified data of the western blots are shown in the corresponding graphs. $\# P<0.05$ and $\# \# P<0.01$, compared to the $\mathrm{CCl}_{4}$-control group; ${ }^{*} P$ $<0.05$ and $* * P<0.01$, compared to the model group. 
Table 1. Contribution scores of the compound combinations

Compound Combination
Contribution

Score 
Figure 1

A

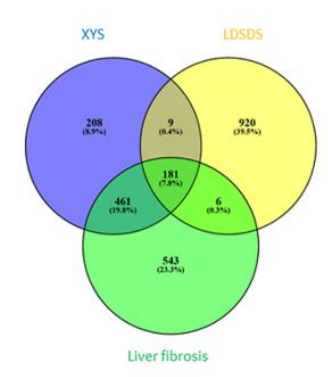

B

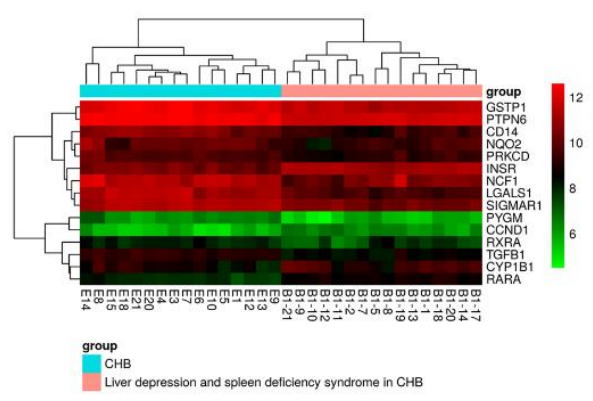

D

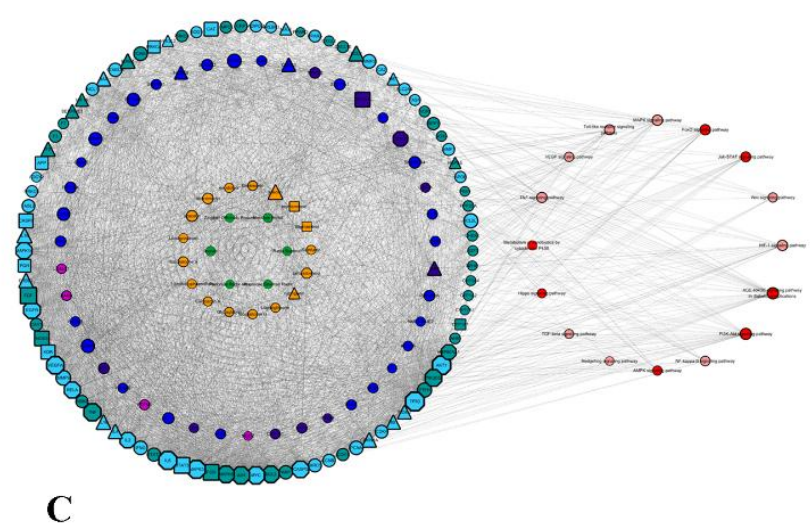

C
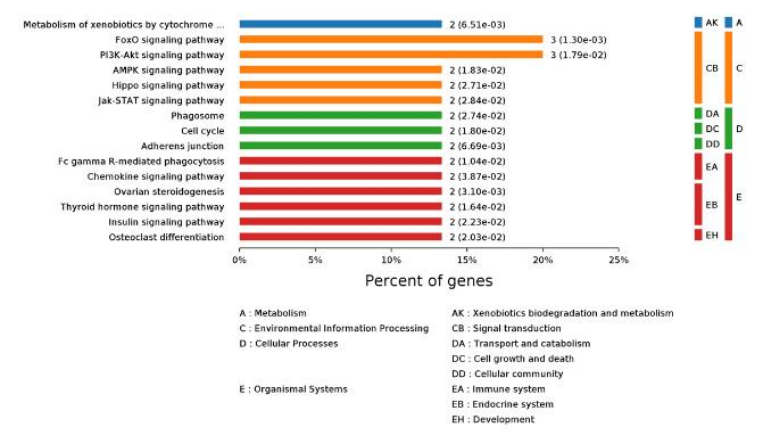
Figure 2

A
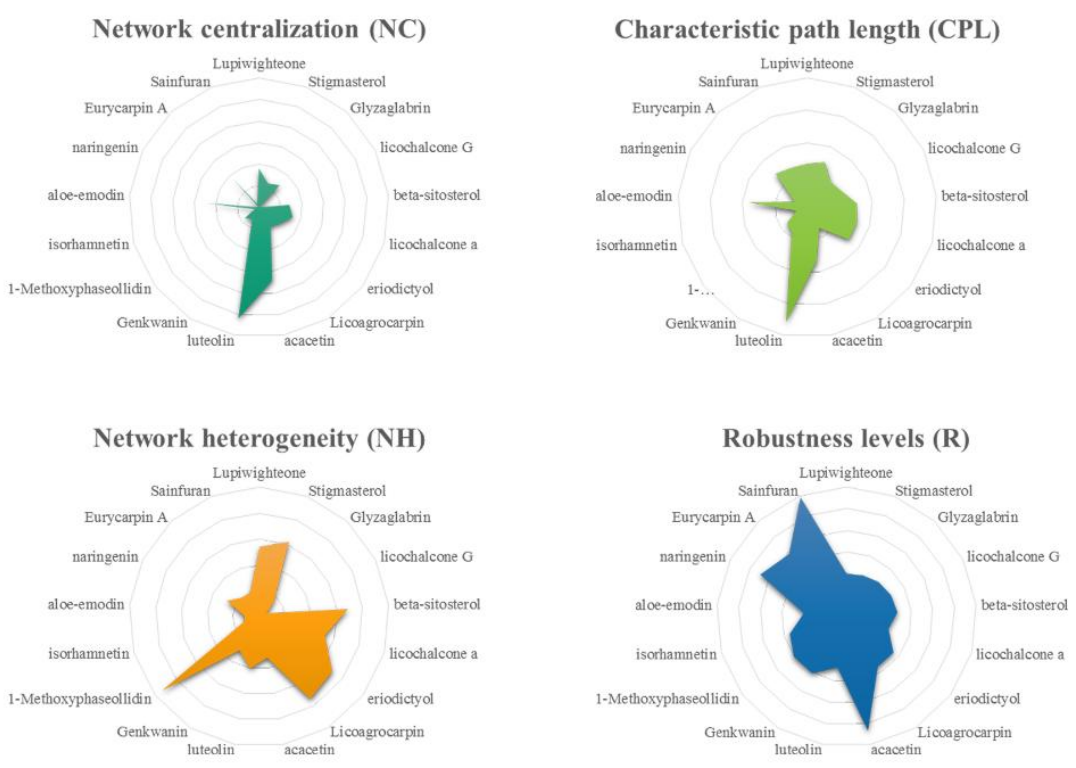

B

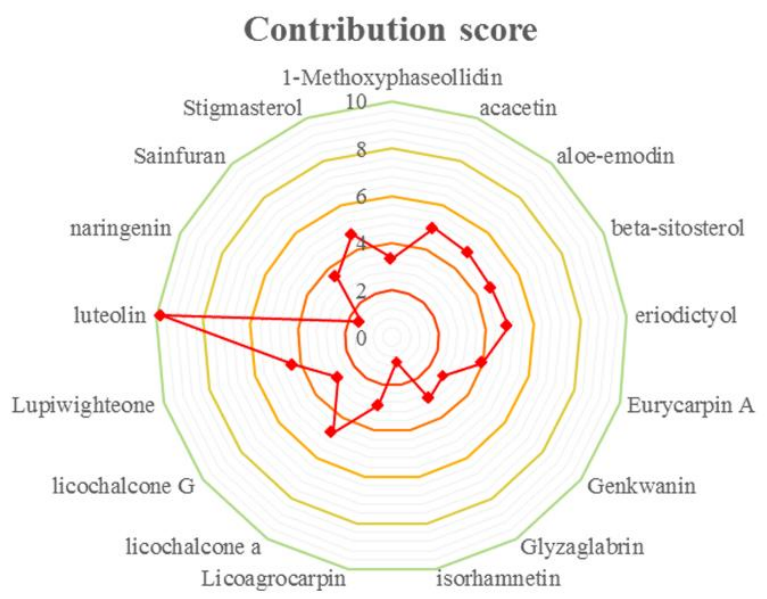




\section{Figure 3}

A
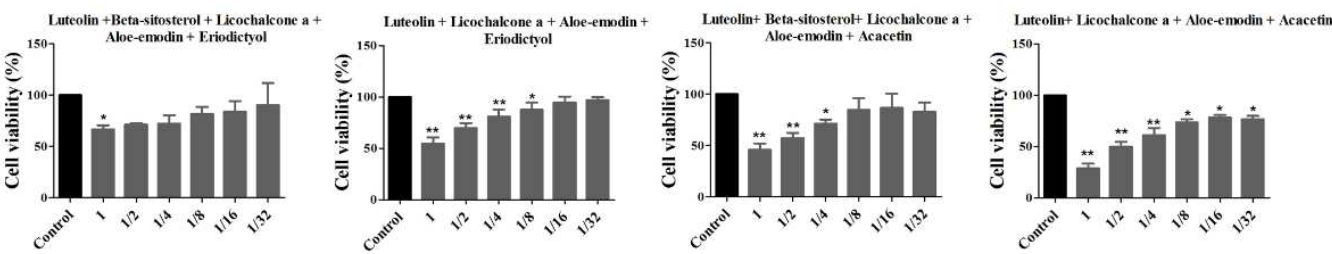

B
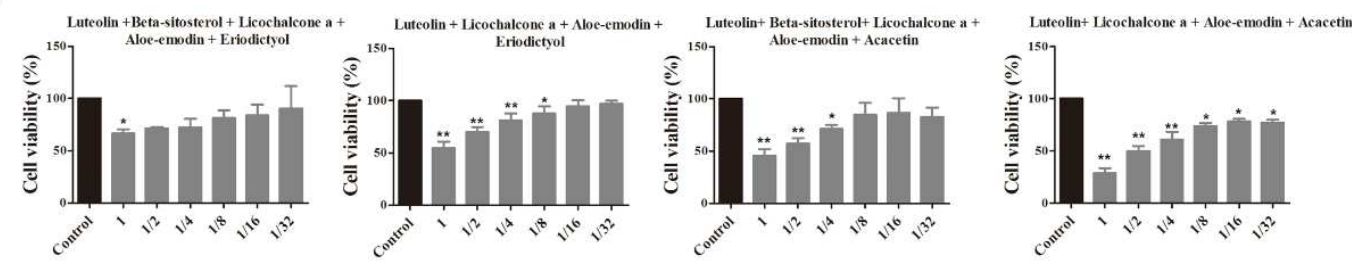

C
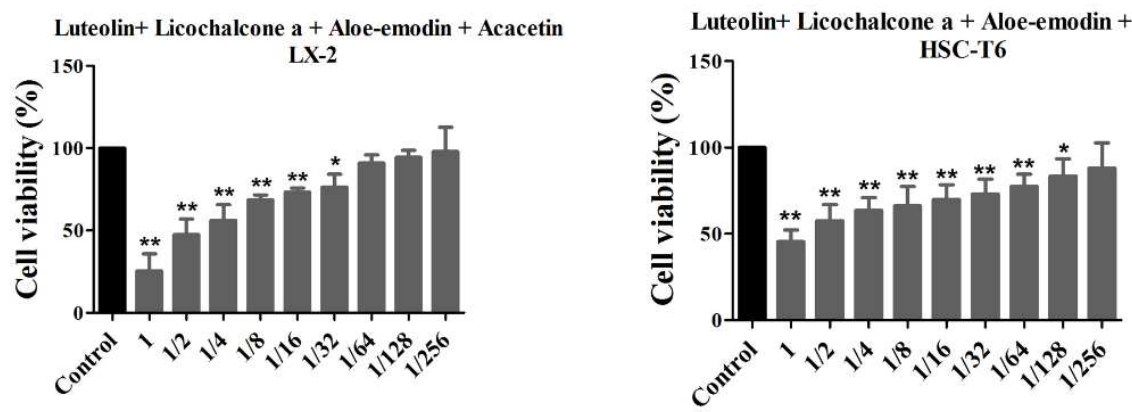


\section{Figure 4}
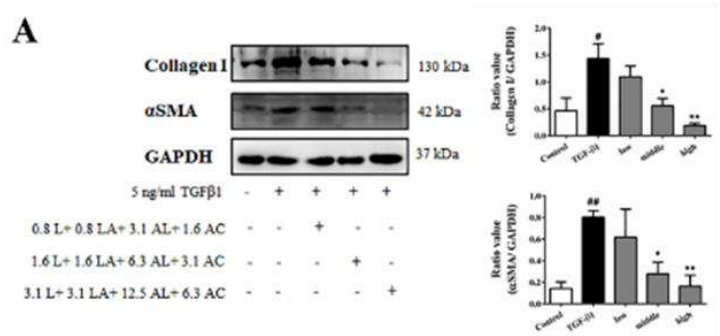

B

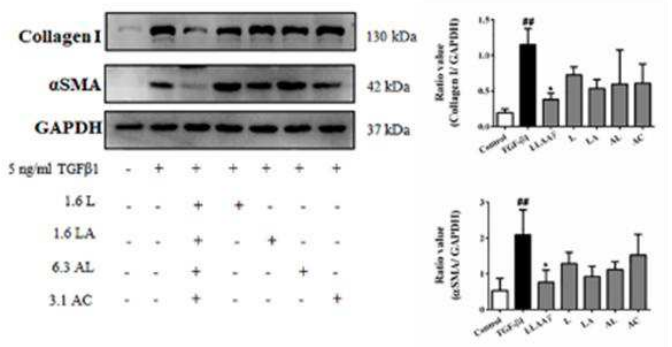

C

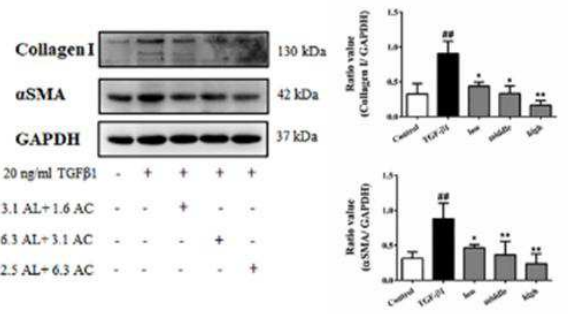

D

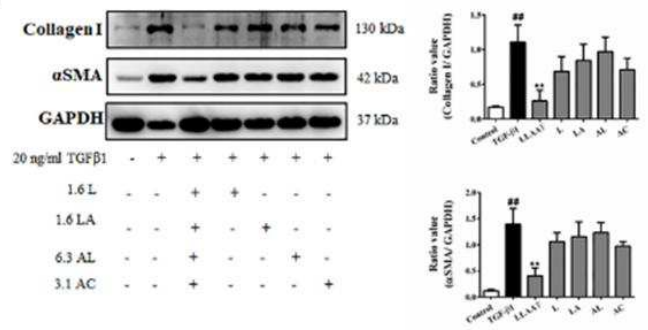




\section{Figure 5}

A
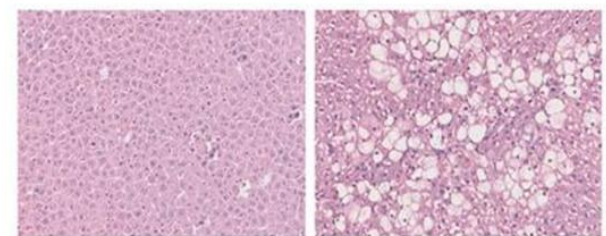

Control

Model

XYS-M

LLAAF

H\&E 200x

B

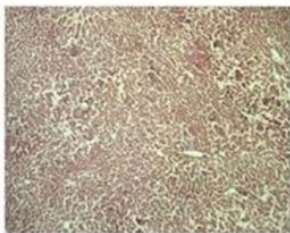

Control

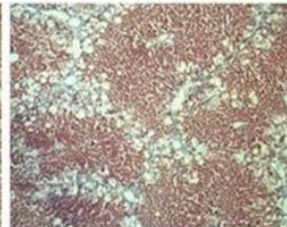

Model

XYS-M

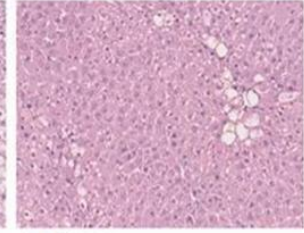

MASSON 100x

C

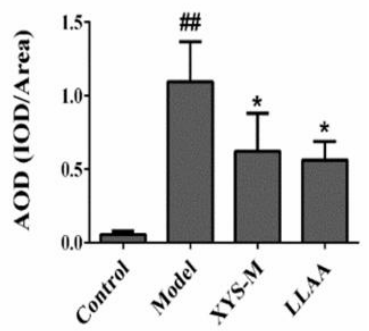

E

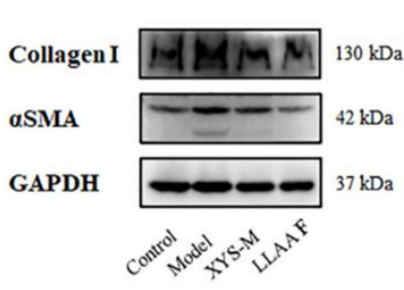

D

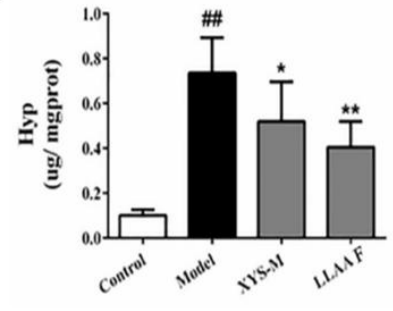

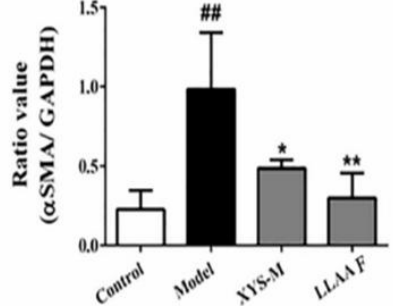


Figure 6

A

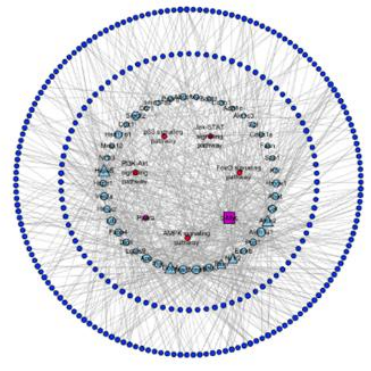

C

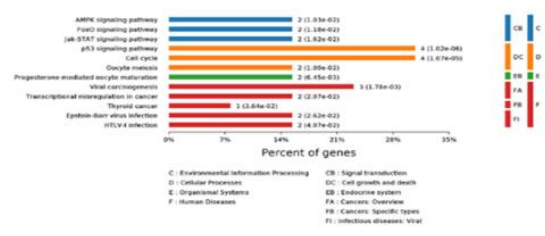

B

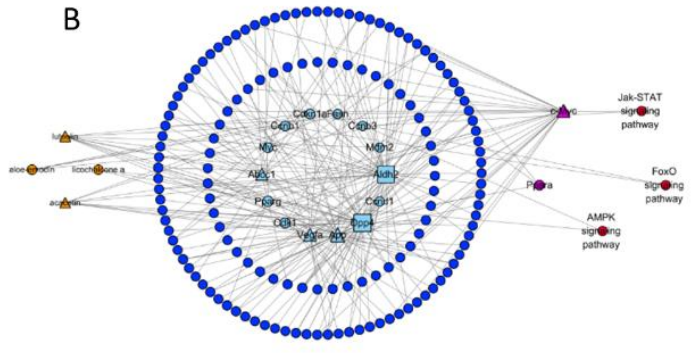

D

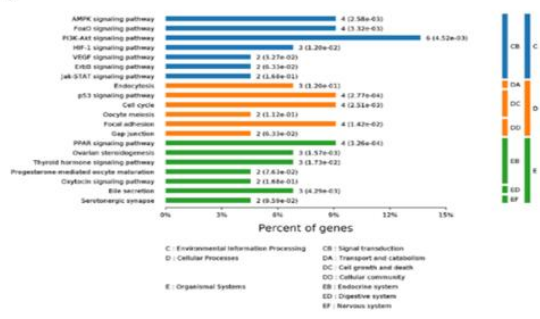

E -log ( $p$-value)

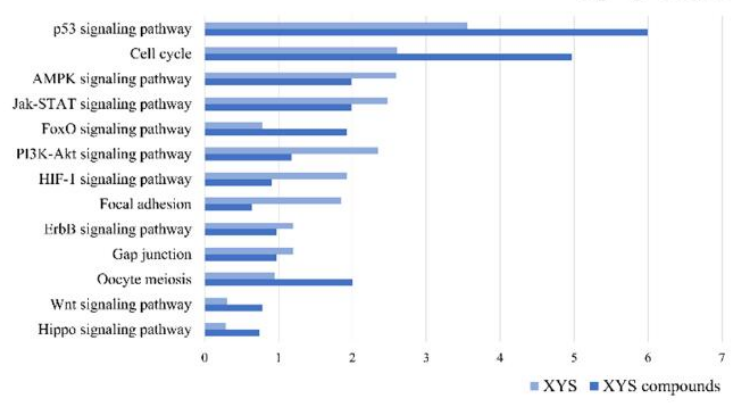


Figure 7

A
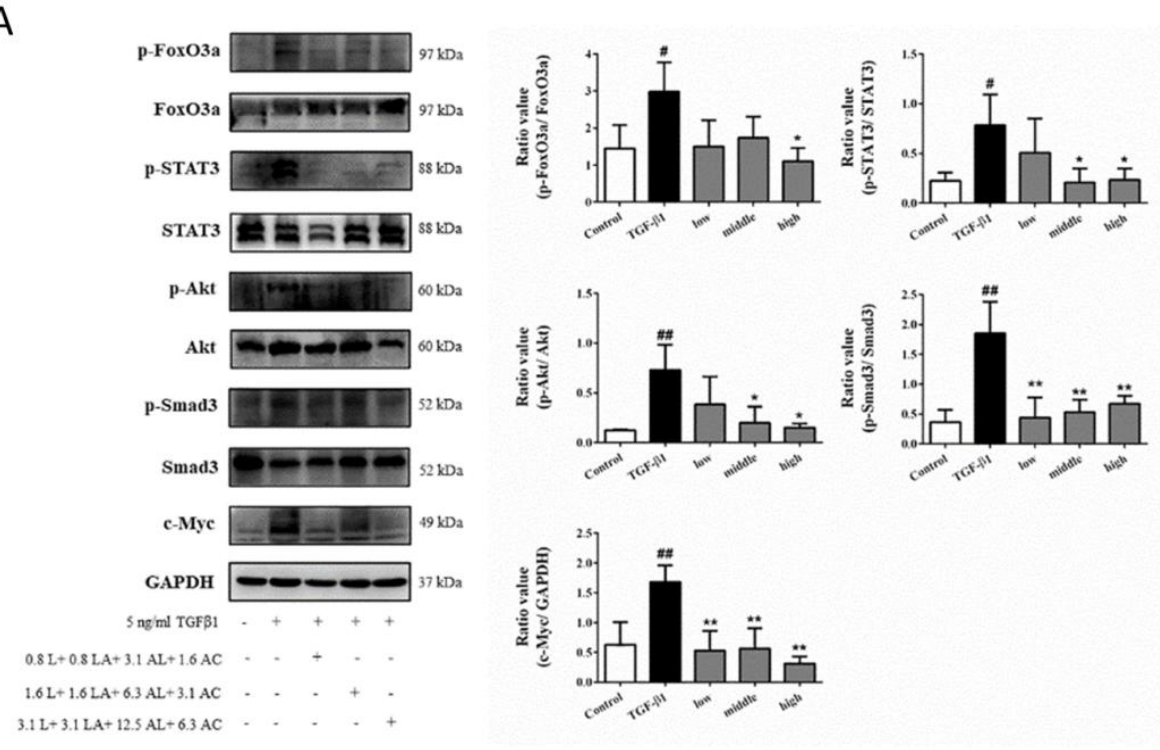

B

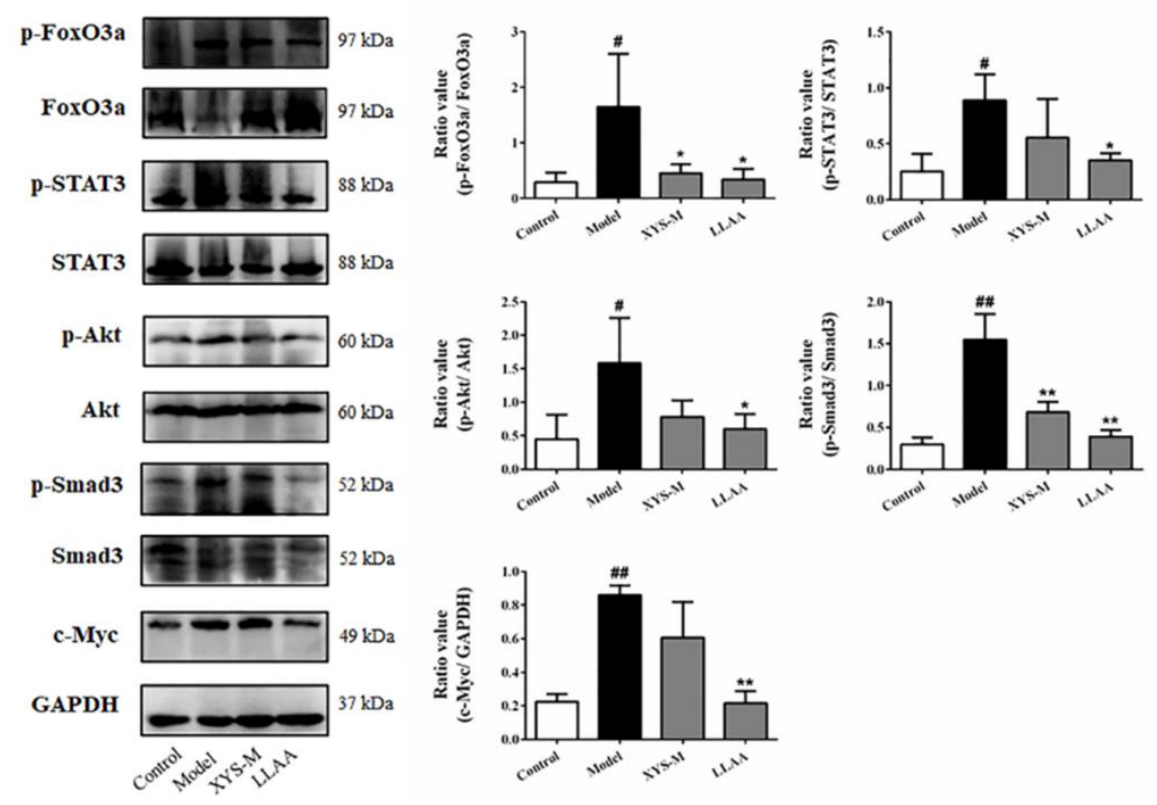




\section{Figures}

A

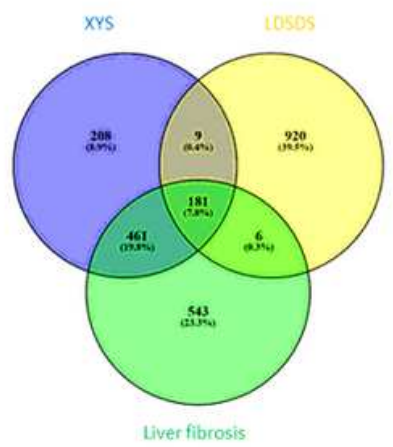

B

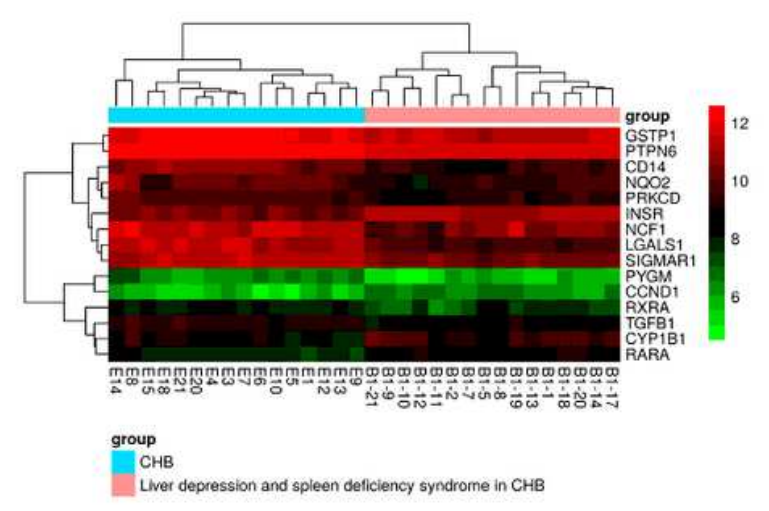

D

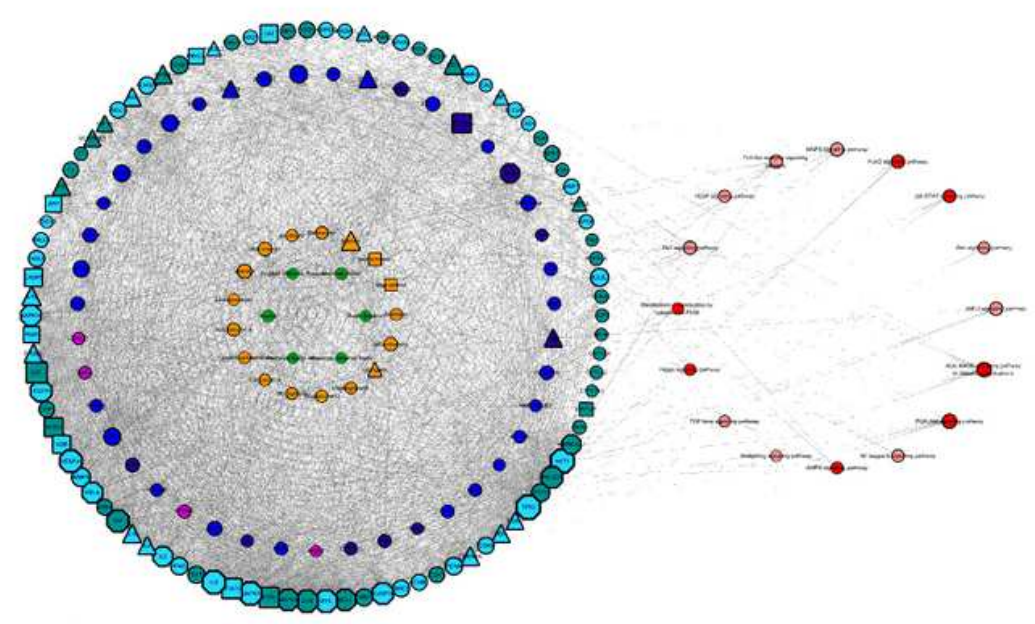

C

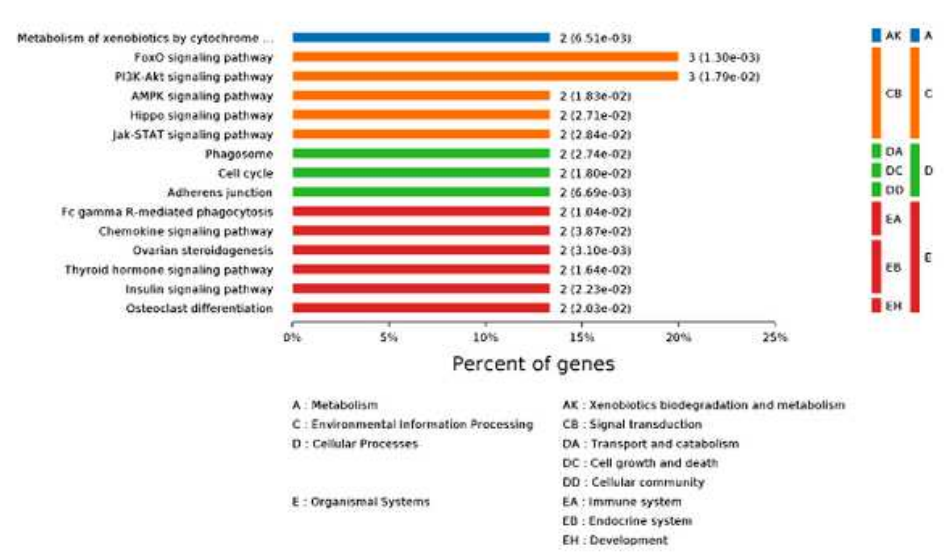

\section{Figure 1}

Identification of the active compounds, gene targets, and signaling pathways of XYS. (A) mRNA microarray analysis of differentially expressed genes (DEGs) in the XYS, LDSDS, and liver fibrosis groups.

(B) Heatmap of DEGs between chronic hepatitis B patients with or without LDSDS ( $n=16$ in each group).

(C) The GO terms of the pathway analysis directly related to XYS. (D) The XYS-LDSDS-liver fibrosis core network analysis. Green, herbs; orange, compounds; indigo, target proteins directly related to XYS; purple, predicted target proteins directly related to XYS; dark blue, target proteins indirectly related to XYS; light blue, target proteins intermediately related to XYS; dark green, predicted target proteins intermediately related to XYS; deep red, core pathway; pink, noncore pathway. (E) The KEGG enrichment analysis of the XYS-LDSDS-liver fibrosis core network. 
A
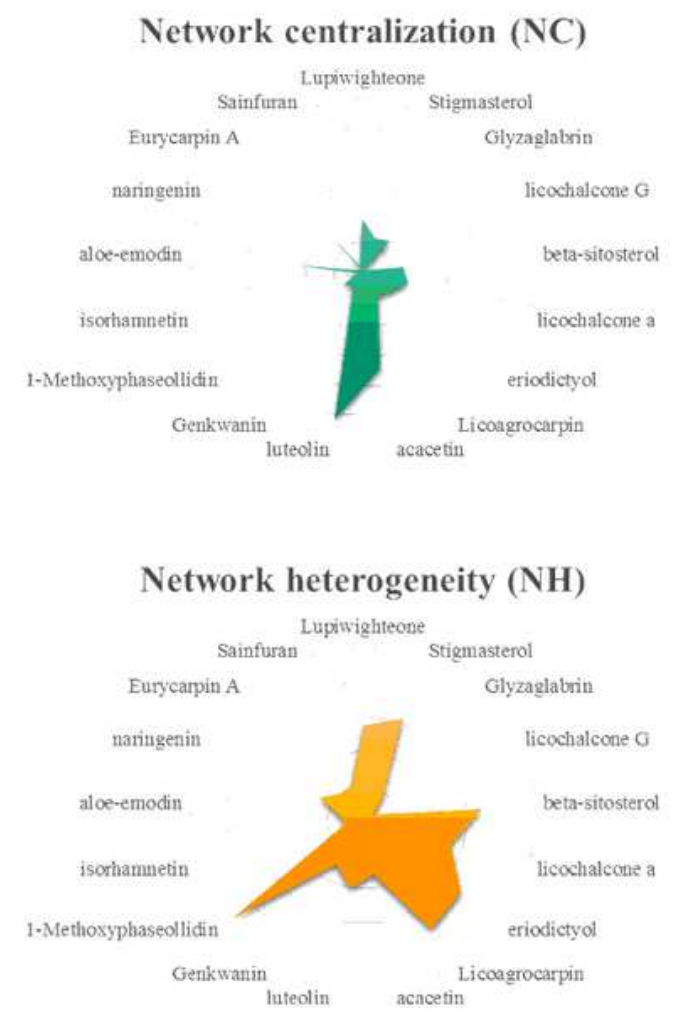

Characteristic path length (CPL)
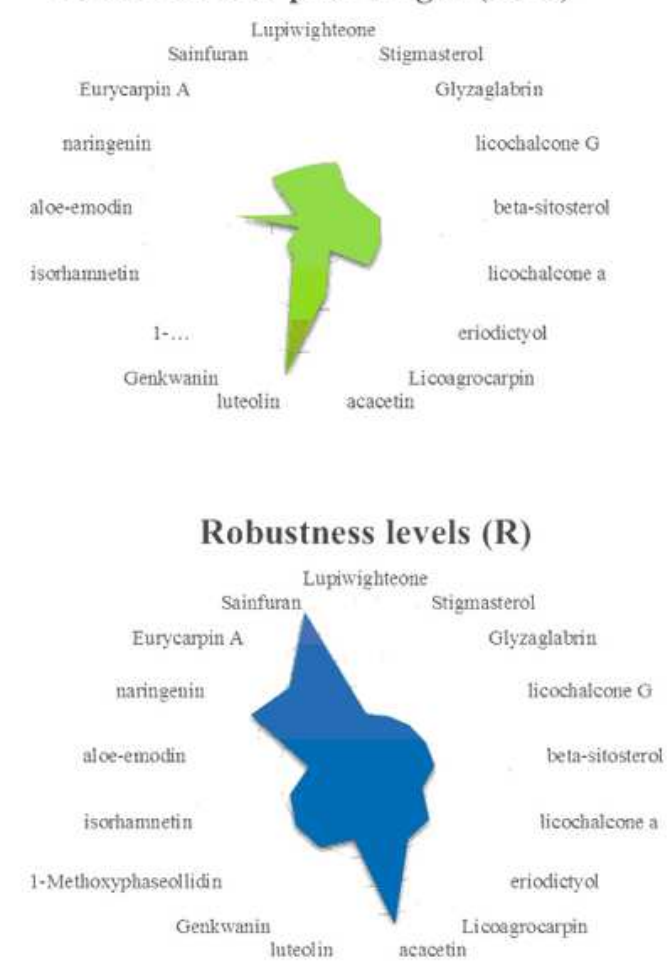

B

\section{Contribution score}

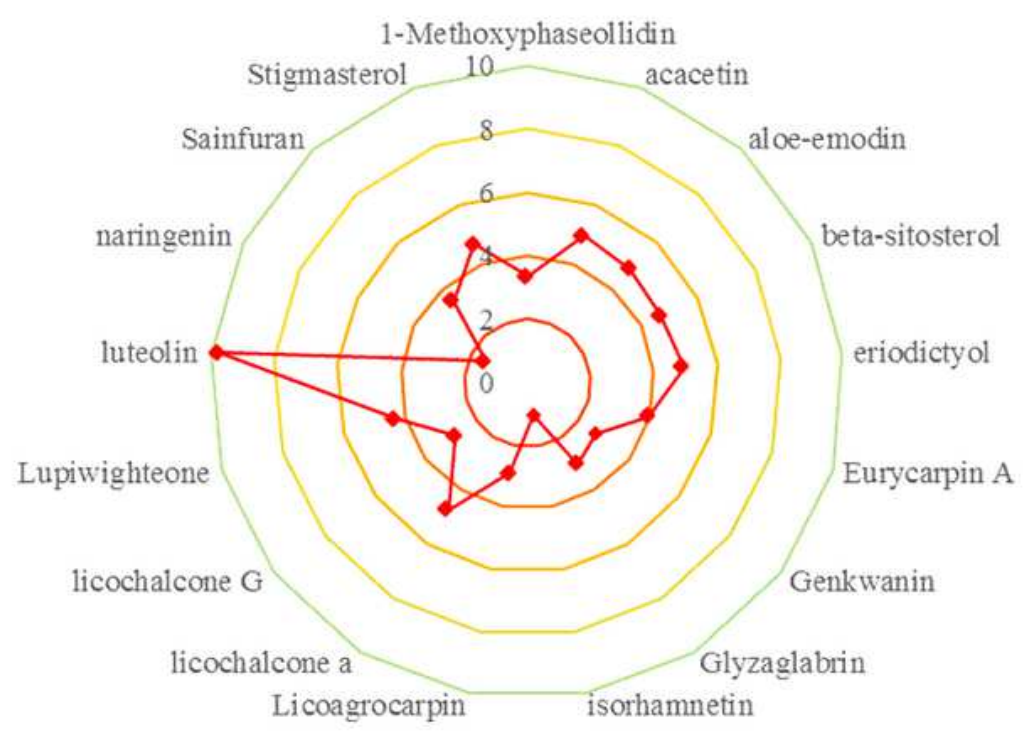

\section{Figure 2}

Contribution score analysis of the XYS-LDSDS-liver fibrosis core network. (A) Network centralization (NC), characteristic path length (CPL), network heterogeneity $(\mathrm{NH})$, and robustness $(\mathrm{R})$ scores of the 17 compounds by using the entropy method and the weighted summation method, which were directly related to the network stability using Cytoscape 3.2.1 software. (B) Analysis of the contribution scores of the 17 compounds by combinations of the NC, CPL, NH and R parameters. 
A
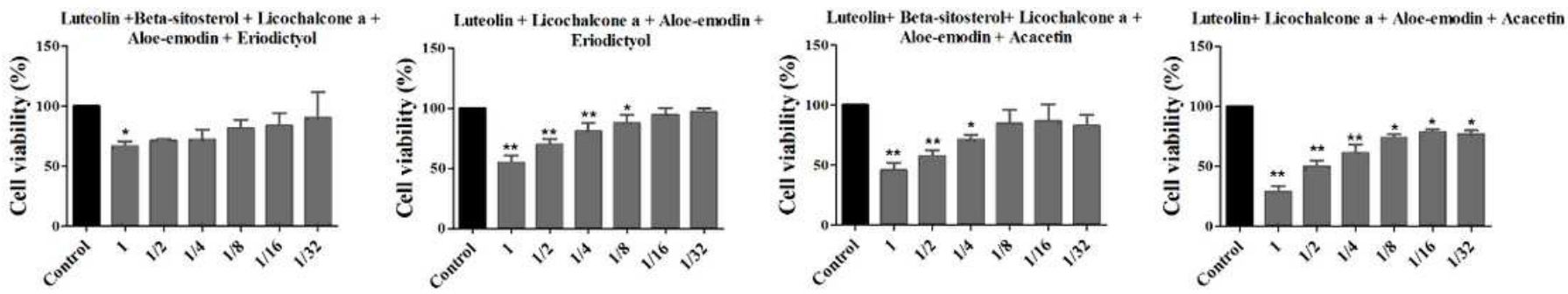

B
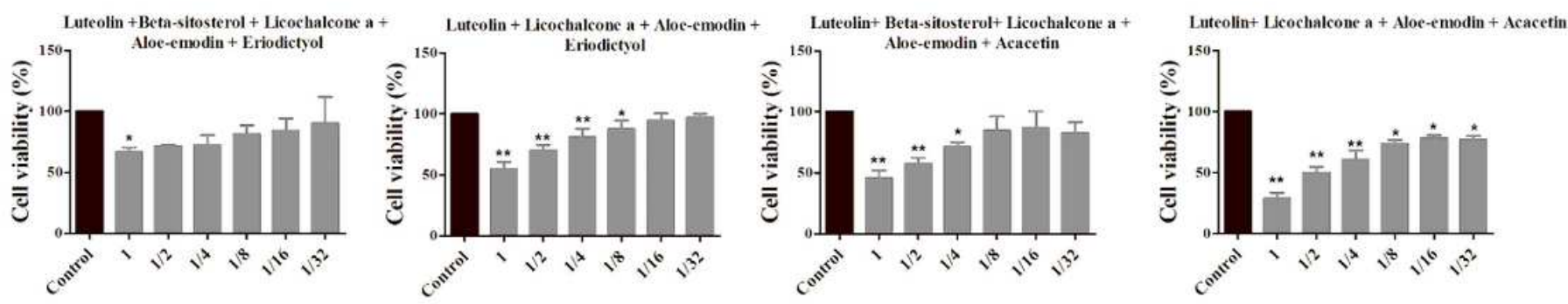

C
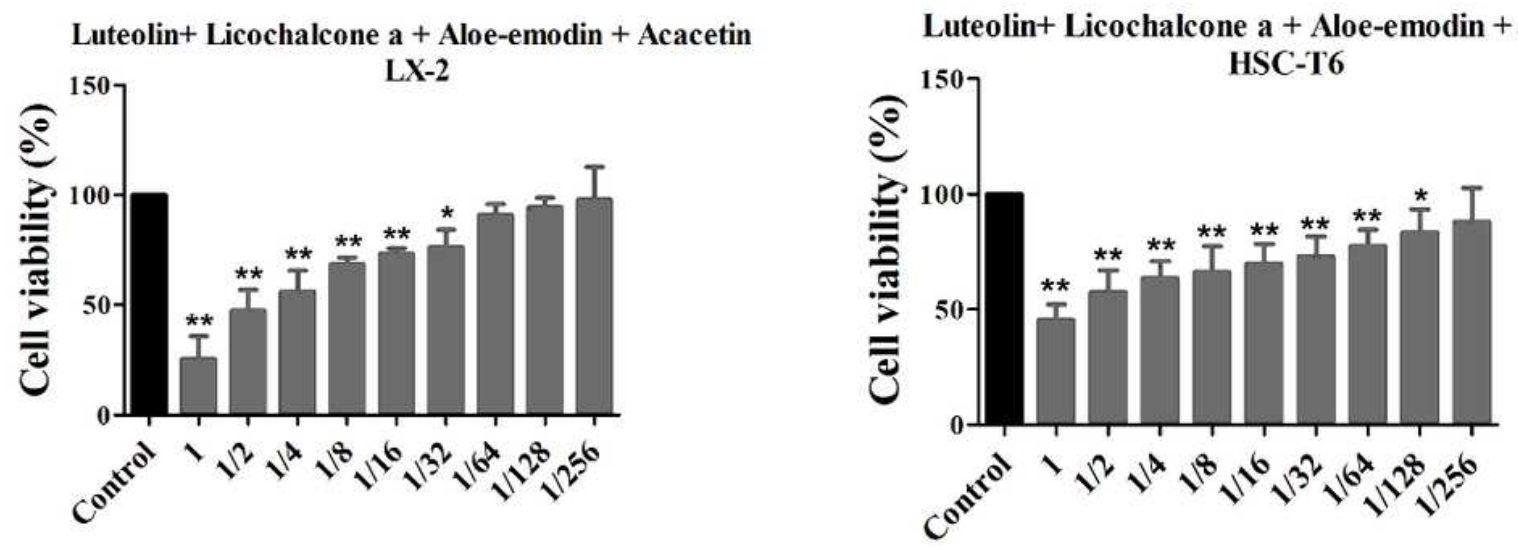

\section{Figure 3}

Effects of six individual XYS compounds and their combinations on regulating cell viability. (A) Cell viability assay. Four top compound combination from network analysis show the effect on the LX2 cells. (B) Cell viability assay. Four top compound combination from network analysis show the effect on the HSC-T6 cells. . ${ }^{*} \mathrm{P}<0.05$ and ${ }^{\star *} \mathrm{P}<0.01$, compared to the control cells. (C) The most active compounds combination in the LX2 and HXC-T6 cells. 
A

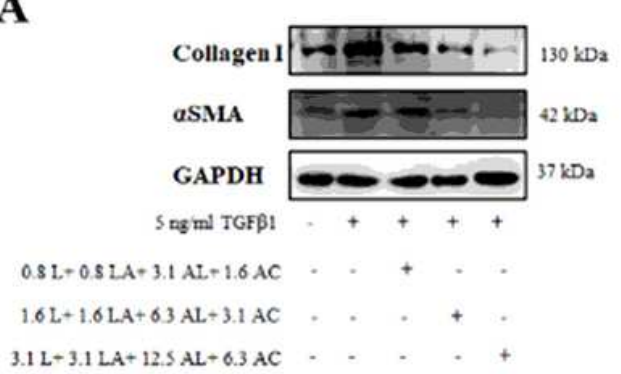

\section{B}

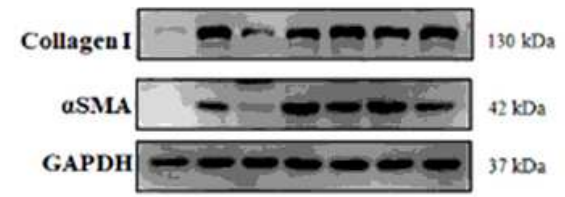

$5 \mathrm{ng} \mathrm{m}$ ! TGFB!

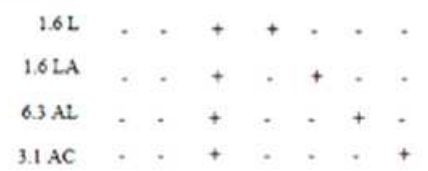

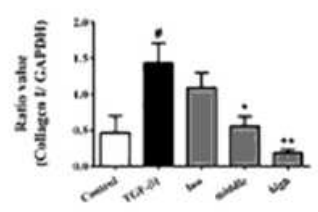
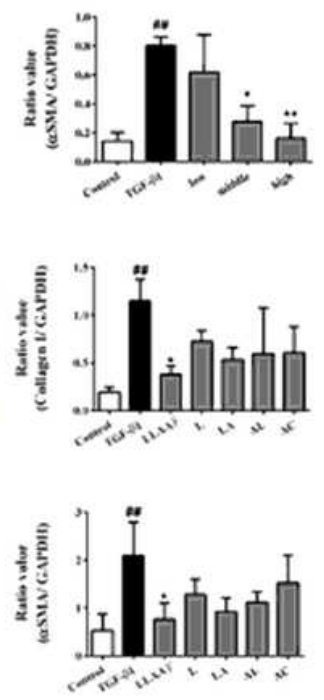

C
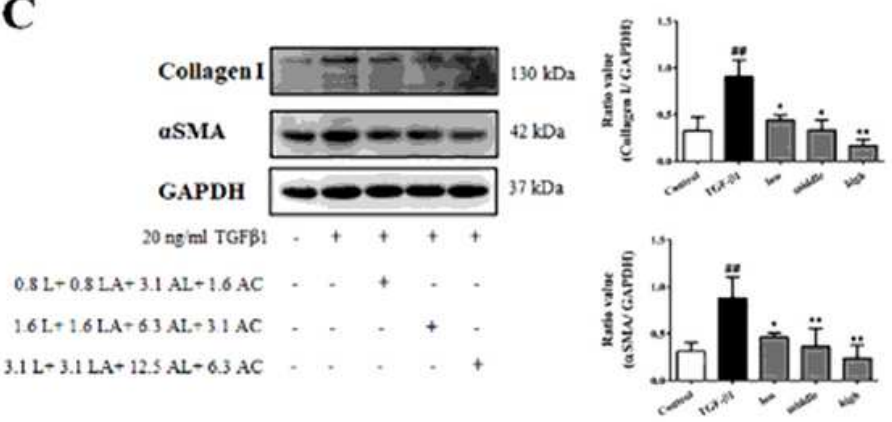

D

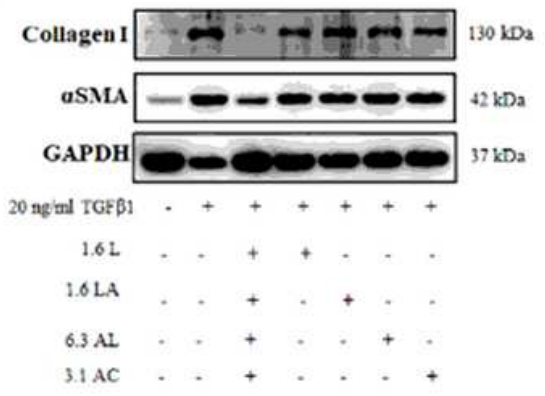

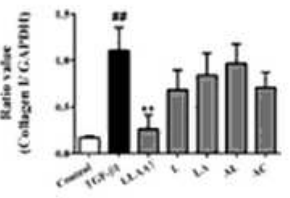

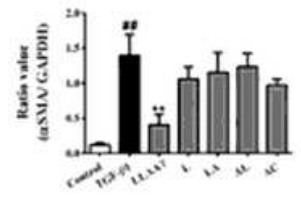

\section{Figure 4}

Effect of LLAAF on regulating protein expression in vitro. (A) Western blot. LX2 cells were grown and treated with a high, medium, or low dose of LLAAF and then subjected to western blot analysis of a-SMA and collagen I expression. Quantified data of the western blots are shown in the corresponding graphs.

(B) Western blot. LX2 cells were grown and treated with a medium dose of LLAAF and its compounds and then subjected to western blot analysis of a-SMA and collagen I expression. (C) Western blot. HSC-T6 cells were grown and treated with a high, medium, or low dose of LLAAF and then subjected to western blot analysis of a-SMA and collagen I expression. (D) Western blot. HSC-T6 cells were grown and treated with a medium dose of LLAAF and its compounds and then subjected to western blot analysis of a-SMA and collagen I expression. Quantified data of the western blots are shown in the corresponding graphs. \#P $<0.05$ and \#\#P $<0.01$, compared to the CCl4-control group; ${ }^{*} \mathrm{P}<0.05$ and ${ }^{*} \mathrm{P}<0.01$, compared to the model group. 
A
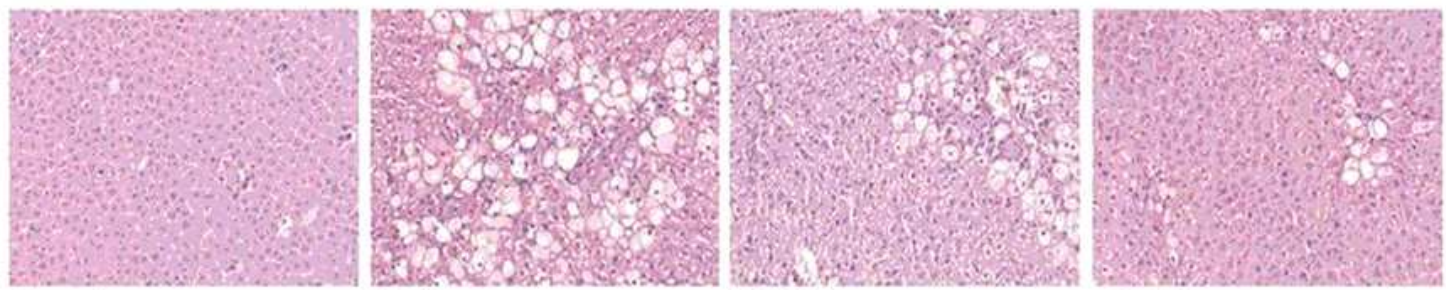

Control

Model

XYS-M

LLAAF

H\&E 200X

B

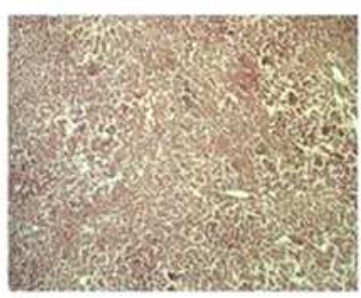

Control

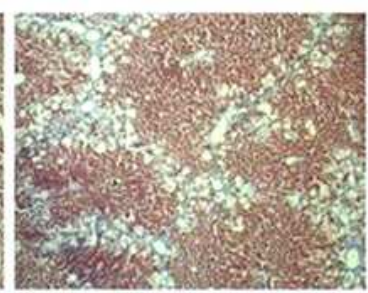

Model

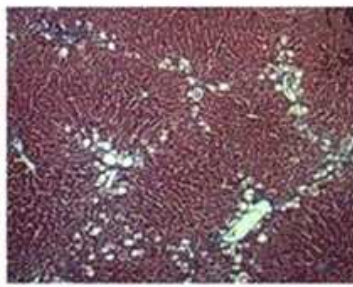

XYS-M

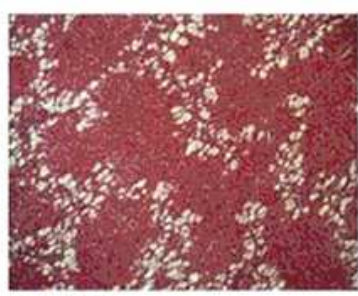

LLAAF

MASSON 100x

C

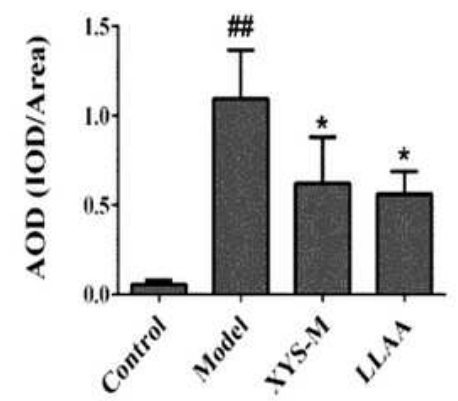

D

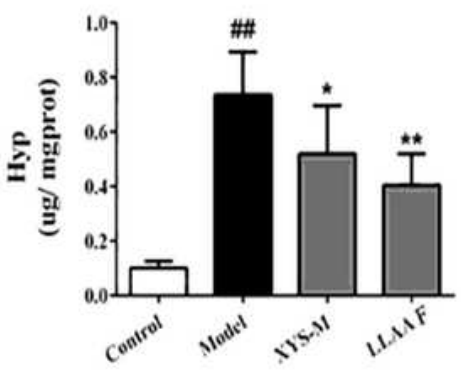

E
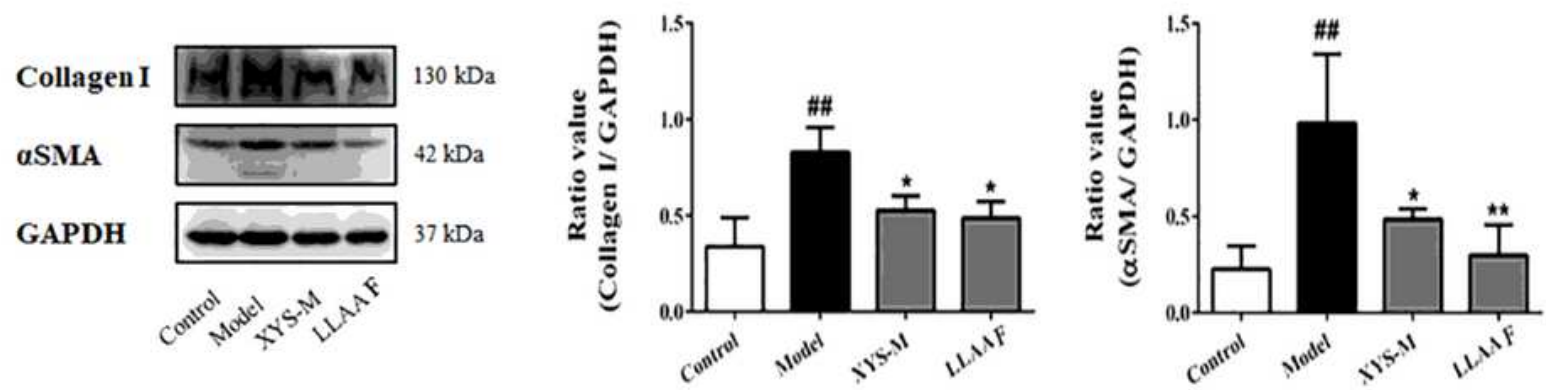

\section{Figure 5}

Effects of XYS-M and LLAAF treatment on reduction of rat liver fibrosis. (A) H\&E staining (200x magnification). (B) Masson's staining (100x). (C) Semi-quantitative analysis of the collagen fibers. (D) The hydroxyproline (Hyp) content. (E) Western blot. The levels of alpha-smooth muscle actin (a-SMA) and collagen I were analyzed using western blot of rat liver fibrotic tissues. \#\#P $<0.01$, compared to the control group; ${ }^{*}<<0.05,{ }^{\star \star} \mathrm{P}<0.01$, compared to the model group. 
A

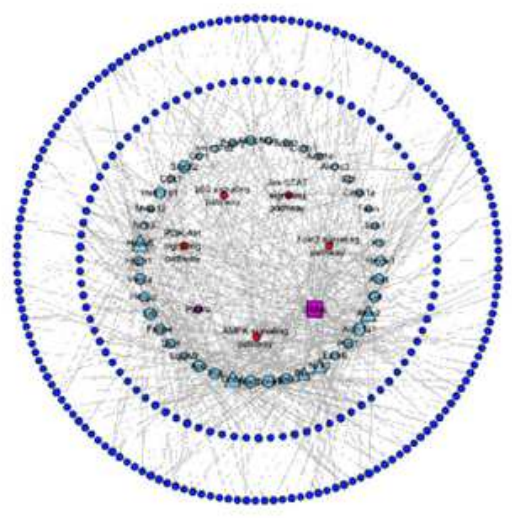

C

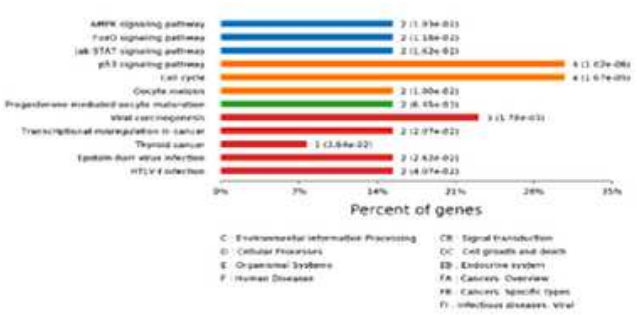

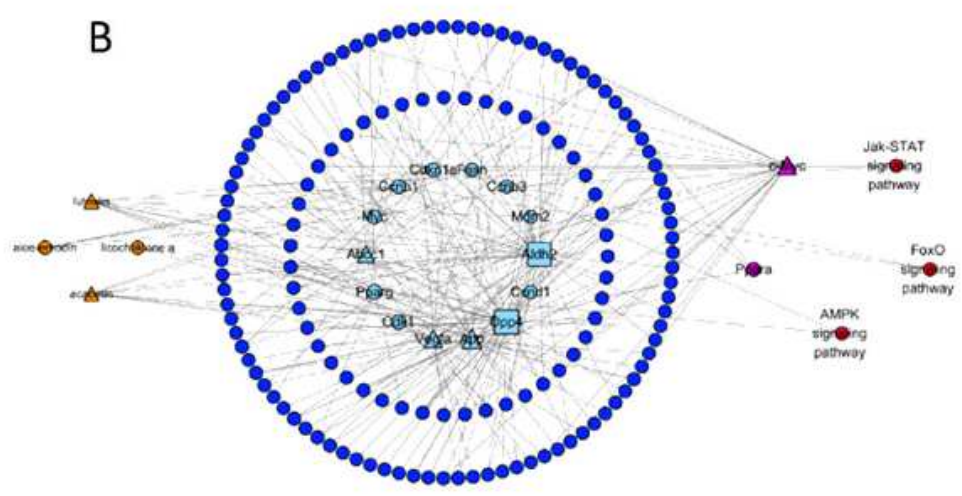

D
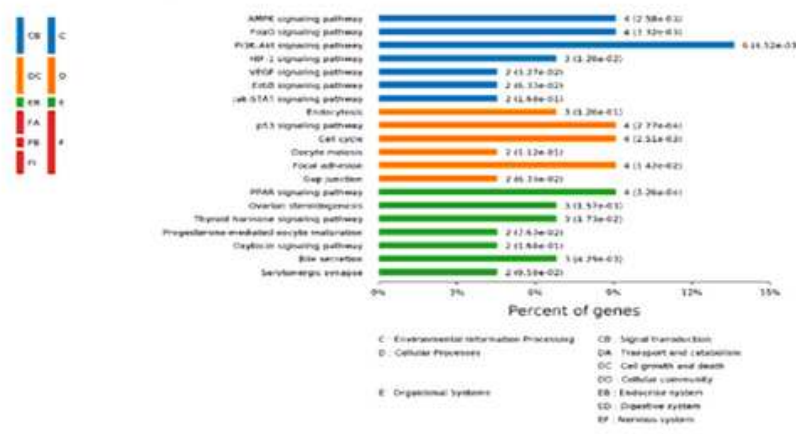

E

-log ( $p$-value)

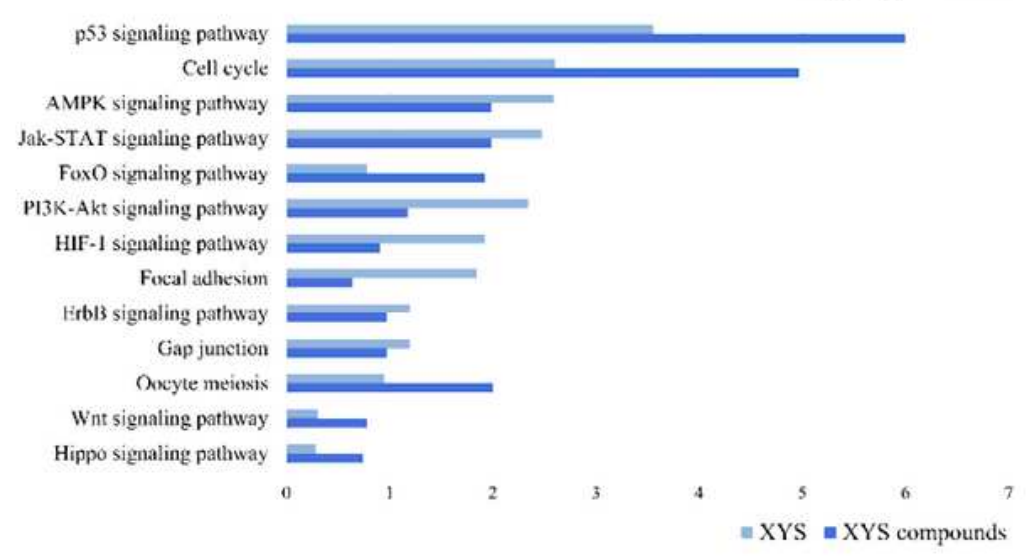

\section{Figure 6}

Bioinformatics analysis of the XYS- and LLAAF-modified PPI networks. (A) XYS network. (B) LLAAF network. (C) KEGG enrichment analysis of the XYS network. (D) KEGG enrichment analysis of the LLAAF network. (E) Pathway analysis between XYS and LLAAF. 
A
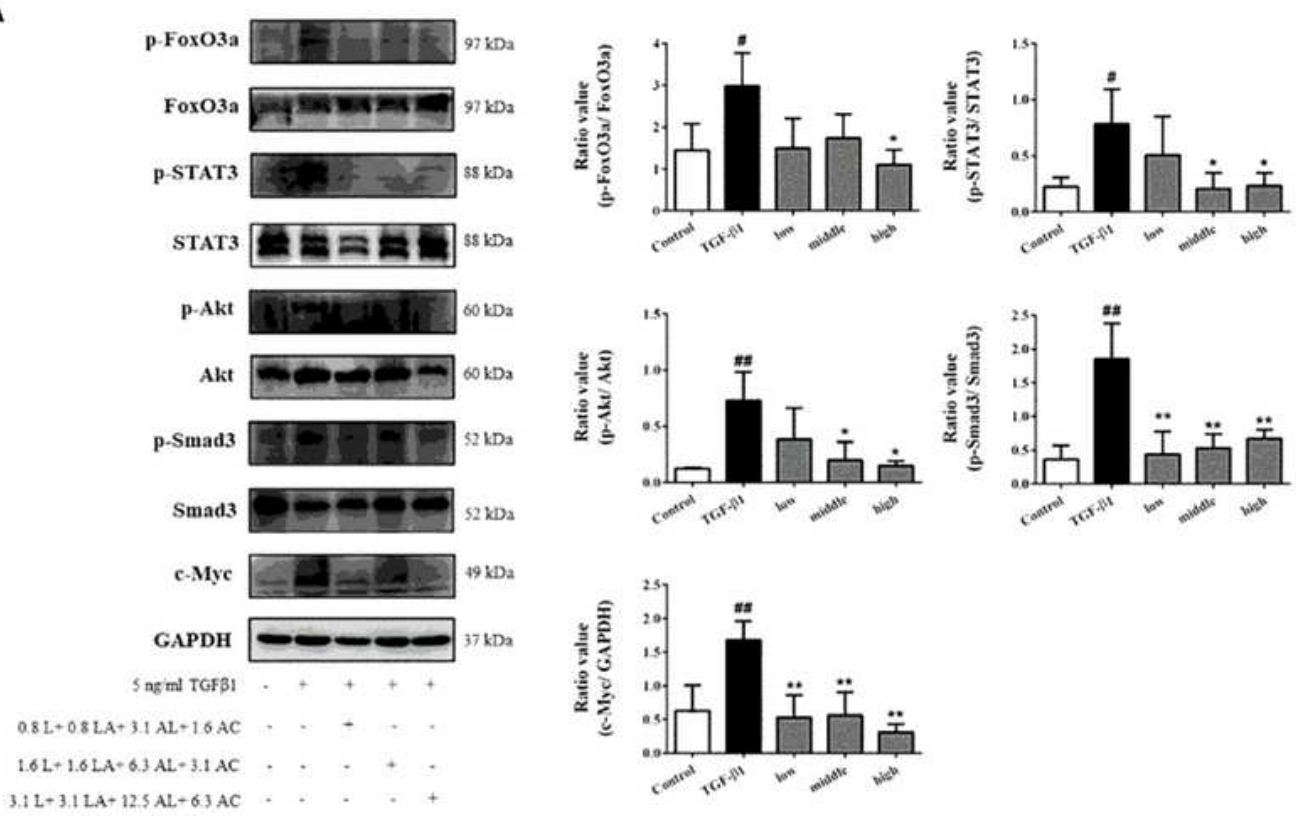

B
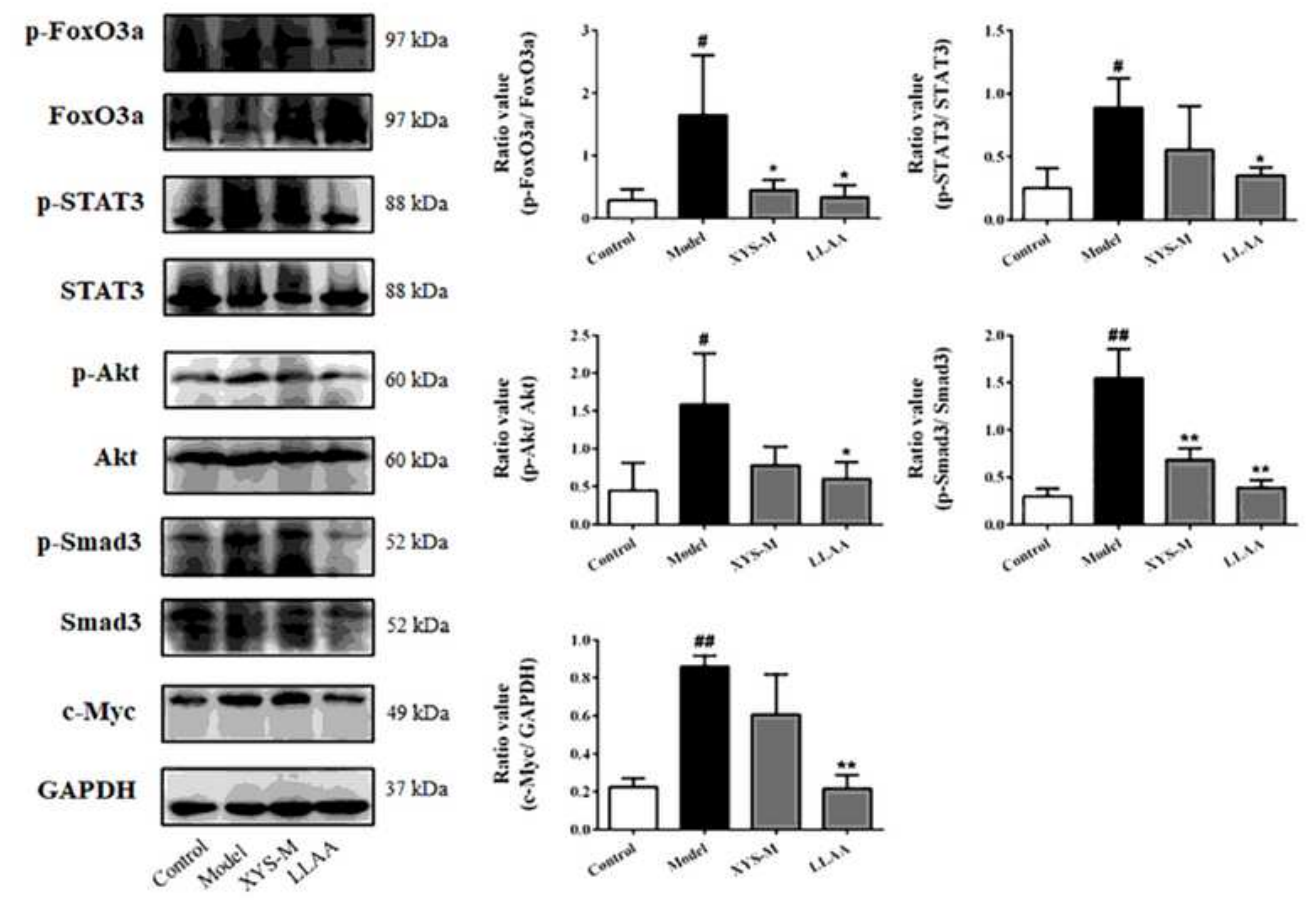

Figure 7

Effect of LLAAF treatment on regulation of Jak-STAT and PI3K-Akt-FoxO signaling. (A) Western blot. LX2 cells were grown, treated with TGF- $\beta 1$ and then with LLAAF for $24 \mathrm{~h}$, and then subjected to western blot analysis of protein expression. (B) Western blot. Liver tissues were resected from rats and subjected to western blot analysis of alpha-smooth muscle actin ( $\mathrm{a}-\mathrm{SMA}$ ) and collagen I expression. Quantified data 
of the western blots are shown in the corresponding graphs. \#P $<0.05$ and \#\#P $<0.01$, compared to the CCl4-control group; $* \mathrm{P}<0.05$ and ${ }^{\star *} \mathrm{P}<0.01$, compared to the model group.

\section{Supplementary Files}

This is a list of supplementary files associated with this preprint. Click to download.

- SupplementaryFigure.docx 